\title{
Regulation of Prefrontal Patterning, Connectivity and Synaptogenesis by Retinoic Acid
}

3

\section{Mikihito Shibata ${ }^{1,+}$, Kartik Pattabiraman ${ }^{1,2,+}$, Belen Lorente-Galdos ${ }^{1}$, David}

6 Andrijevic ${ }^{1}$, Xiaojun Xing ${ }^{1,3}$, Andre M. M. Sousa ${ }^{1}$, Gabriel Santpere ${ }^{1,8}$, and Nenad

Sestan ${ }^{1-7,9, *}$

1Department of Neuroscience, Yale School of Medicine, New Haven, CT 06510, USA ${ }^{2}$ Yale Child Study Center, New Haven, CT 06510 ${ }^{3}$ Yale Genome Editing Center, Yale School of Medicine, New Haven, CT 06510, USA ${ }^{4}$ Department of Comparative Medicine, Yale School of Medicine, New Haven, CT 06510, USA ${ }^{5}$ Department of Genetics, Yale School of Medicine, New Haven, CT 06510, USA ${ }^{6}$ Department of Psychiatry, Yale School of Medicine, New Haven, CT 06510, USA ${ }^{7}$ Program in Cellular Neuroscience, Neurodegeneration and Repair, CT 06510, USA ${ }^{8}$ Neurogenomics Group, Research Programme on Biomedical Informatics, Hospital del Mar Medical Research Institute, Department of Experimental and Health Sciences, Universitat Pompeu Fabra, Barcelona, Catalonia, Spain ${ }^{9}$ Kavli Institute for Neuroscience, Yale University, New Haven, CT 06520

\section{+These authors contributed equally to this work}

\section{*Correspondence to Nenad Sestan (nenad.sestan@yale.edu)}




\section{Abstract}

34 The prefrontal cortex (PFC) and its reciprocal connections with the mediodorsal

35 thalamus (MD) are crucial for cognitive flexibility and working memory ${ }^{1-4}$ and are

36 thought to be altered in several disorders such as autism spectrum disorder ${ }^{5,6}$

37 and schizophrenia ${ }^{6-9}$. While developmental mechanisms governing regional

38 patterning of the rodent cerebral cortex have been characterized ${ }^{10-15}$, the

39 mechanisms underlying the development of PFC-MD connectivity and the lateral

40 expansion of PFC with distinct granular layer 4 in anthropoid primates ${ }^{16-23}$ have

41 not been elucidated. Here we report increased concentration of retinoic acid (RA),

42 a signaling molecule involved in brain development and function ${ }^{24,25}$ in the

43 prospective PFC areas of human and macaque, compared to mouse, during mid-

44 fetal development, a crucial period for cortical circuit assembly. In addition, we

45 observed the lateral expansion of RA synthesizing enzyme, ALDH1A3,

46 expression in mid-fetal macaque and human frontal cortex, compared to mouse.

47 Furthermore, we found that enrichment of RA signaling is restricted to the

48 prospective PFC by CYP26B1, a gene encoding an RA-catabolizing enzyme

49 upregulated in the mid-fetal motor cortex. Gene deletion in mice revealed that RA

50 signaling through anteriorly upregulated RA receptors, $R \times r g$ and $R$ arb, and

51 Cyp26b1-dependent catabolism is required for the proper molecular patterning

52 of PFC and motor areas, the expression of the layer 4 marker RORB, intra-PFC

53 synaptogenesis, and the development of reciprocal PFC-MD connectivity.

54 Together, these findings reveal a critical role for RA signaling in PFC

55 development and, potentially, its evolutionary expansion. 
Introduction

66 The proper specialization and expansion of higher-order processing or association 67 areas in the cerebral cortex, specifically PFC, is thought to underlie complex cognitive 68 capabilities. The PFC reaches greatest complexity in anthropoid primates, which have 69 many prefrontal areas that cover the entire anterior two-thirds of the frontal lobe with a 70 well-defined or granular layer $4^{16,17,19-23}$. Mice and rats, the most commonly studied 71 rodents, have fewer prefrontal areas, which are located within the medial agranular 72 (lacking obvious layer 4) frontal cortex that are closest in homology to the primate 73 agranular medial PFC ${ }^{18,21}$. Given the anatomical and functional divergence of the PFC 74 between primates and rodents, understanding specification of the expanded primate 75 PFC, which likely underlies some of the most distinctly human or primate aspects of 76 cognition, requires analysis of the developing primate cortex. Our previous analyses of 77 the developing human brain revealed that the transcriptomic differences between neocortical areas are transient and most prominent during mid-fetal development ${ }^{26,27}$, a crucial period for specification and the initial assembly of neocortical neural circuits ${ }^{28}$.

80 Thus, we hypothesized that the molecular processes governing the developmental 81 specification of the human PFC could be revealed by differential regional gene expression analysis of the mid-fetal human neocortex.

Prefrontal-enriched gene expression gradient in the mid-fetal human neocortex

85 To identify molecular processes governing the developmental specification of human 86 PFC, we screened for genes that are upregulated in the mid-fetal frontal lobe, using 87 tissue-level RNA-sequencing (RNA-seq) data from BrainSpan and PsychENCODE projects $^{27}$. These data comprise mid-fetal samples from eleven prospective neocortical areas ranging in age from 16 to 22 postconception weeks (PCW), including four PFC

90 areas (medial, mPFC/MFC; orbital, oPFC/OFC; dorso-lateral, dIPFC/DFC; and ventro91 lateral, vIPFC/VFC) and the primary motor cortex (M1C). Gene expression in these 92 frontal lobe areas was compared to areas within the parietal (primary sensory cortex, 93 S1C; and inferior parietal cortex, IPC), occipital, (primary visual cortex, V1C) and 94 temporal lobes (primary auditory cortex, A1C; superior temporal cortex, STC; and 95 inferior temporal cortex, ITC) (Fig. 1a; Extended Data Fig. 1a). We identified 190 96 protein-coding genes that are specifically upregulated in at least one area within a lobe 
97 in comparison with areas from other lobes: 125 in the frontal lobe, 46 in the temporal 98 lobe, 17 in the parietal lobe, and 2 in the occipital lobe (Extended Data Fig. 1a,b). The expression profiles of these 190 differentially expressed genes were able to differentiate

100 the four brain lobes and most regions within them, as observed by principal component 101 analysis (PCA). Moreover, the first principal component (PC1), the one accounting for 102 the highest variability present in the data, corresponded to the anterior-posterior axis of 103 the neocortex with genes specifically upregulated in the frontal lobe mainly negatively 104 correlated with PC1 while genes upregulated in the other lobes are positively correlated 105 with PC1 (Fig. 1d). Interestingly, Gene Ontology (GO) enrichment analysis of the 125 106 genes upregulated in the mid-fetal frontal lobe identified a significant enrichment of 107 genes associated with categories such as response to retinoic acid (RA), synapse 108 development and axon development/guidance (Fig. 1c; Supplementary Table 1), 109 suggesting that these genes may play a role in frontal lobe patterning and circuit 110 development. Accordingly, multiple frontal lobe upregulated genes associated with RA 111 signaling, such as CBLN2, RXRG, CBLN1, NEUROD1, NEUROG2, CDH8, CYP26A1, 112 and MEIS2 24,25,29-31 (Supplementary Table 2; see also this and our accompanying 113 study ${ }^{31}$ ), were amongst those with the highest impact on PC1 (Fig. 1d).

114 As the frontal lobe consists of both prefrontal association and motor regions, we 115 checked whether RA-related genes were upregulated in the PFC as compared to the 116 prospective M1C. Analyzing only the RNA-seq samples encompassing the five areas 117 of the mid-fetal human frontal lobe from Fig. $1 \mathrm{a}^{27}$, we identified 116 protein-coding genes 118 upregulated in at least one of the four prospective PFC areas and 190 genes 119 upregulated in the prospective M1C. Together, these 306 differentially expressed genes 120 distinguished M1C from PFC as shown by PCA (Fig. 1e). RA-related genes, such as 121 CBLN2, RXRG, CDH8, MEIS2, and RBP1 were among the genes upregulated in the 122 PFC compared to the M1C, while the RA degrading enzyme CYP26B1 was upregulated 123 in the M1C compared to the PFC (Fig. 1f; Supplementary Table 2), consistent with 124 our previous microarray-based findings in the human mid-fetal cortex ${ }^{32}$. Upregulated 125 expression of RXRG and CBLN2 in prospective mid-fetal PFC were confirmed by in situ 126 hybridization in human as well as in rhesus macaque, the most commonly studied non127 human anthropoid primate, and mouse, at developmental ages equivalent to the human 128 mid-fetal period ${ }^{28}$ (Extended Data Fig. 2c; see also our accompanying study ${ }^{31}$ ). We 
129 also identified multiple autism spectrum disorder (ASD) risk genes, such as

130 CACNA2D3, CDH8, CNTNAP2, and MEGF10 (Group 1-3 from SFARI Gene database, 131 gene.sfari.org), upregulated in the mid-fetal PFC (Fig. 1f; Supplementary Table 2).

\section{Prefrontal-enriched retinoic acid concentration gradient in mid-fetal primates}

$134 \mathrm{RA}$ is a diffusible biologically active derivative of vitamin A previously implicated in 135 neurogenesis, differentiation and synaptic function of cortical neurons $24,25,29,30,33-38$. RA 136 is also known to exert a trophic influence on nascent neurons to promote axon and 137 dendritic outgrowth ${ }^{33,35,39,40}$ as well as synaptogenesis ${ }^{35,41}$. Moreover, alterations in RA 138 signaling have been implicated in the pathophysiology of ASD ${ }^{42-44}$ and schizophrenia ${ }^{45-}$ 139 48. Given the enrichment of RA-related genes among those upregulated in the mid-fetal 140 human PFC, we assessed whether RA concentration is increased in the PFC, using an 141 RA-specific enzyme-linked immunosorbent assay (ELISA) in two anthropoid primates 142 with laterally expanded PFC, human and macaque, and mouse, a rodent with fewer, 143 medially located, PFC areas. Quantification of RA concentration in the same eleven 144 mid-fetal neocortical areas analyzed for differential gene expression revealed a 145 prominent PFC-enriched anterior-posterior gradient of RA concentration in postmortem 146 human $(16,18,18,19$ PCW; $N=3-4$ brains per areal measurement) and macaque (four 147 brains at 110 postconceptional days (PCD); $\mathrm{N}=3-4$ brains per areal measurement) 148 neocortex, with mPFC exhibiting the highest concentration within each species (Fig. 149 2a). Overall, RA concentrations were much higher in prospective PFC areas (mPFC, 150 dIPFC, oPFC and vIPFC) compared to more posterior areas (M1C, S1C, IPC, V1C, 151 A1C, STC, and ITC; Two-tailed unpaired t-test: $P=2 e-6 ; N=15$ in PFC areas, 21 in 152 posterior areas). Complementary analysis of mouse neonatal cortex revealed higher 153 RA concentrations in prospective mPFC compared to the adjacent secondary and 154 primary motor areas ( $\mathrm{P} 1 ; \mathrm{N}=3$ brains; Fig. $2 \mathbf{2 a})$.

155 Comparison across the three species at approximately equivalent 156 developmental age, identified higher concentrations of RA in both the human mPFC 157 and all four PFC areas overall as compared to the other two species (Two-tailed 158 unpaired t-test: Human all PFC vs. macaque all PFC: 1.45 fold change, $P=0.02$; 159 human mPFC vs macaque mPFC: 1.45 fold change, $P=0.03$; human all PFC vs mouse 160 mPFC: $F C$ 6.48, $\mathrm{P}=3 \mathrm{e}-8$; human mPFC vs mouse mPFC: 9.34 fold change, $\mathrm{P}=$ 
161 0.0005; Fig. 2a), as well as in macaque compared to mouse (Unpaired t-test: Macaque 162 all PFC vs mouse mPFC: 4.48 fold change, $\mathrm{P}=2 \mathrm{e}-6$; macaque $\mathrm{mPFC}$ vs mouse mPFC, 1636.45 fold change, $P=0.003$; Fig. 2a). Interestingly, ITC, an association area within the 164 temporal lobe thought to exhibit unique features and connectivity in humans ${ }^{23,49}$, had a 165 higher RA concentration among non-frontal areas in humans but not in macaque 166 (Unpaired t-test: $P=0.01$; Fig. 2a).

Lateral expansion of the mid-fetal expression of retinoic acid synthesizing enzyme in primates

Next, we assessed how RA concentration differs across the medial-lateral axis of the mid-fetal frontal lobe in human and macaque compared to the mouse frontal cortex at

173 the medial frontal cortex, which comprises PFC areas (mPFC), compared to adjacent 174 dorso-lateral regions corresponding to prospective secondary and primary motor areas 175 (Fig. 2a). The ratio of the concentration of RA between the dorsal and medial $176 \mathrm{PFC/frontal}$ cortex was elevated in human and macaque compared to mouse, with no 177 significant difference between human and macaque (Two tailed ratio paired t-test: 178 human vs. mouse, $P=0.003$; macaque vs. mouse, $P=0.003$; human vs. macaque, 179 NS; Fig. 2b), indicating that RA signaling is expanded laterally in the human and 180 macaque mid-fetal frontal cortex, compared to mouse.

The observed enrichment of RA in the PFC led us to examine the mid-fetal expression of a key RA synthesizing enzyme, ALDH1A3/RALDH3 24,25,53 in human, macaque and mouse. The mPFC enrichment of RA concentration in all three species (Fig. 2a, b), closely overlaps with previously reported expression of ALDH1A3 in mice ${ }^{54}$. We confirmed this in the neonatal mouse frontal cortex ( $N=3$ brains) and found Aldh1a3 187 the cingulate cortex (Fig. 2c). However, unlike the medially restricted expression of 188 Aldh1a3 in mice, we identified low but spatially broader expression of human ALDH1A3 189 (21 and 22 PCW; $\mathrm{N}=2$ brains) throughout the cortical plate, underlying subplate zone 190 and white matter in all regions of the anterior part of the mid-fetal frontal lobe, which 191 correspond to the prospective areas of the medial, orbital and lateral PFC (Fig. 2c). A 192 similar pattern of regional expression was observed throughout the cortical plate of the 
macaque cortex (114 and 140 PCD; $N=2$ brains), but with less noticeable expression

194 in the subplate zone and white matter, compared to human PFC (Fig. 2c). This finding indicates that the expression domain of $A L D H 1 A 3$ has expanded dorso-laterally and ventro-laterally in human and macaque mid-fetal frontal lobe, similar to lateral extension of granular PFC in anthropoid primates ${ }^{16,20}$.

Developmental prefrontal retinoic acid signaling is mediated by RXRG and RARB Given the mid-fetal anterior cortical upregulation of both RA and ALDH1A3, we further assessed the expression of RA-dependent receptors and RA-responsive downstream genes in the human, macaque and mouse cortex. In addition to Rxrg, which is upregulated in the human mid-fetal PFC (Fig. 1f and Johnson et al. ${ }^{32}$ ), we also identified an upregulation of Rarb in the P0 mouse anterior cortex by quantitative PCR (Extended Data Fig. 2a,b). In situ hybridization revealed that both $R X R G$ and $R A R B$ orthologs are more highly expressed in the striatum as compared to the cerebral cortex. However, within the mid-fetal human (21, 22 PCW; $N=2$ brains) and macaque (114, 140 PCD; $\mathrm{N}=2$ brains) cortex, or neonatal mouse cortex (P0; $\mathrm{N}=3$ brains), the two genes exhibited a high anterior to low posterior gradient of expression (Extended Data Fig. 2c).

The RXRG/RARB heterodimer has been previously shown to mediate RA

212 signaling in the adult mouse cerebral cortex and striatum, and be required for learning,

213 locomotion and dopamine signaling ${ }^{29,30}$. To assess whether RXRG and RARB are 214 required for RA signaling activity in the developing mouse frontal cortex, we generated 215 constitutive Rxrg and Rarb double knockout (dKO) mice (Extended Data Fig. 2d), 216 which, consistent with previous findings ${ }^{29,30}$, are viable. To assess RA signaling in these $217 \mathrm{dKO}$ mice, we crossed these mice with the RARE-lacZ reporter line ${ }^{52}$ where lacZ is 218 under the transcriptional control of a RA response element (RARE). Using control 219 RARE-lacZ mice we first confirmed previously described enrichment of RA signaling in 220 the dorsal portion of medial frontal and limbic areas in neonatal mouse ${ }^{51}$ (Fig. 3a;

221 Extended Data Fig. 3a). We then identified a reduction of RA signaling in the neonatal 222 dKO mPFC (Two-tailed Student's t-test: WT vs. dKO, $P=1 e-6$ in section 1; $P=0.0003$ 223 in section 2 in Fig. 3a, Extended Data Fig. 3a). In addition, we found a less extensive 224 reduction of RA signaling in the anterior cingulate area (ACA) and retrosplenial area at 
225 P0 (Extended Data Fig. 3a). We also observed reduction of RA signaling in the

226 hippocampus and outer shell of the striatum of dKO mice (Extended Data Fig. 3a).

227 There was no reduction of signaling in the thalamus, where expression was restricted

228 to ventroposterior nuclei (Extended Data Fig. 3a). Furthermore, expression of genes

229 upregulated in the human mid-fetal and mouse neonatal frontal lobe and known to be

230 regulated by $\mathrm{RA}^{31,54}$, including Cbln2 and Meis2 (Fig. 1e), were reduced in the dKO

231 mice (Extended Data Fig. 3b). These findings indicate that RXRG and RARB are

232 required for $\mathrm{RA}$ signaling in the neonatal mouse mPFC.

233

234 RXRG and RARB regulate prefrontal synaptogenesis and axon development

235 To understand the functional significance of RA signaling through the RXRG/RARB

236 heterodimer in the developing cortex, we performed RNA-seq analysis of different

237 regions/areas of the P0 mouse frontal cortex (i.e., mPFC; secondary motor cortex

238 (MOs) and the adjacent parts of the primary motor cortex (MOp); and OFC, as indicated

239 in Fig. 2c) microdissected from postmortem Rarb and Rxrg dKO and WT littermate

240 control brains. We identified 4768 differentially expressed (DEx) protein-coding genes

241 between the two genotypes in at least one of the areas (Extended Data Fig. 4a). The

242 highest number of DEx genes was found in the mPFC, as expected based on the medial

243 frontal enrichment of RA signaling in P0 mice (mPFC: 2630 genes; OFC: 2095 genes;

244 MOs/MOp: 1240 genes; Extended Data Fig. 4a; Supplementary Table 3). PCA based

245 on the expression of these DEx genes separated the WT and dKO along PC1, with the

246 mPFC showing greatest distance between the WT and the $\mathrm{dKO}$, further supporting the

247 notion that the mPFC was most affected by the reduction of RA signaling (Fig. 3b,

248 Extended Data Fig. 4b).

249 The GO enrichment analysis revealed that terms associated with genes that are

250 overexpressed in the WT frontal cortex compared to the dKO frontal cortex were highly

251 related to the process of synaptogenesis and cellular components related to synapses

252 and axons, whereas the ones overexpressed in the dKO are related to the regulation of

253 the cell cycle (Fig. 3c; Extended Data Fig. 4c,d). In addition, reflecting the enrichment

254 of RA signaling in the medial frontal cortex (Fig. 3c), when analyzing DEx genes in

255 different regions/areas of the frontal cortex, only genes overexpressed exclusively in 256 the mPFC were associated with the cellular components, axons and synapses 
257 (Extended Data Fig. 4c,d). Of note, the majority of DEx genes with GO terms related 258 to axon and synapse development showed the presence of RA receptor binding sites 259 in their associated regulatory elements (promoters, putative enhancers) with genes with 260 reduced expression in the dKO more likely to have at least one RA receptor binding 261 sites (Extended Data Fig. 5). We extended this analysis to the human mid-fetal frontal 262 lobe upregulated genes related to axons and synapses, and identified the presence of 263 RA receptor binding sites in their associated regulatory elements (Extended Data Fig. 264 5). However, binding site enrichment analyses using two independent methods did not 265 reveal over-representation of RA receptor binding sites compared to random sequences 266 or transcriptional start site's flanking regions of human genes for either set of genes 267 (Extended Data Fig. 5). Furthermore, several of the genes downregulated in the dKO 268 related to axon guidance (such as Chl1, Epha8, Efnb3, Epha4, Ephb6, Ntf3, Robo1, 269 Nr4a2, Plxnc1, and Plxnd1) and synapse development (such as Cbln2, Cdh8, and 270 Wnt7b) displayed an anterior-enrichment in WT neonatal mouse cortex (Extended 271 Data Fig. 6a; Supplementary Table 4; see also our accompanying study ${ }^{31}$ ), similar to 272 gradient of Rxrg and Rarb (Extended Data Fig. 2c).

273 We also observed a significant enrichment of orthologous genes specifically 274 upregulated in the human mid-fetal frontal lobe (from Fig. 1) in the list of genes that 275 were downregulated in the dKO mice, including those linked to synapse and axon 276 development, such as Cbln1, Cbln2, Cdh8, Nrp1, Pcdh17, Sema3c and Tnc (Extended 277 Data Fig. 4e; Fig. 1d). Furthermore, downregulated genes identified exclusively in the 278 mPFC were significantly enriched for both orthologous genes specifically upregulated 279 in the human frontal lobe and ASD-related genes (Extended Data Fig. 4e). Finally, 280 genes upregulated exclusively in the OFC were significantly enriched for both ASD-and neuroticism-related genes (Extended Data Fig. 4e).

Since the role of RA in proliferation has been previously investigated $24,25,33$, we 283 analyzed the role of RA in synaptogenesis by quantifying synapse number in multiple 284 regions of the mouse PO cortex between dKO mice and WT littermate controls, and 285 identified a significant reduction of PSD-95-positive excitatory synapses in the mPFC 286 (27.6\% reduction) and MOs (32.4\% reduction), but not in the OFC, primary motor area 287 (Mop), and primary somatosensory area (SSp) (Two-tailed Student's t-test: WT vs. 288 dKO: $P=0.0006$ for mPFC; $P=0.002$ for $M O s ; P=0.09$ for $O F C ; P=0.7$ for $M O p ; P=$ 

specific role for RA signaling in the regulation of synaptogenesis and, possibly, axon development, particularly in the mPFC.

We further investigated the role of RA signaling on long-range connections from the mPFC using Diffusion Tensor Imaging (DTI). Various tracing studies have identified connections to the contralateral mPFC, thalamus, nucleus accumbens, and basolateral amygdala as the main output from the mPFC ${ }^{1-4}, 21$. We identified a profound reduction in long-range connections between the mPFC and thalamus in dKO compared to WT mice at P5 (Paired t-test: WT vs. dKO: $P=0.001$; Fig. 4b). There was no difference in connections between the left and right mPFC at P5 (Fig. 4c). Due to limitations of the technique and underdeveloped axon pathways at this age, we were unable to study the connections between the mPFC and nucleus accumbens, or basolateral amygdala using DTI. Our attempts to use DTI to investigate connections at later ages were hindered by technical air bubble artifacts that arose during preparation of older brains that we were not able to solve in time for this submission.

Thus, we then performed anterograde axon tracing experiments at $\mathrm{P} 21$, where a fluorescent lipophilic dye Dil was placed in the MPFC or medial thalamus (Fig. 4d,e; Extended Data Fig. 6) both to confirm the DTI finding and assess if the reduced mPFCMD connectivity persists at older ages. When tracer was placed in the mPFC, there was a selective loss of labelled processes in two nuclei in the thalamus, likely the mediodorsal (MD) and anteromedial (AM) nucleus in dKO mice compared to controls (67\% reduction, Two-tailed Student's t-test: WT vs. dKO: $P=0.009)$, as well as a

313 reduction of fibers in the internal capsule (Fig. 4d; Extended Data Fig. 6c). Placement

314 of lipophilic tracer in the medial thalamus showed reduction of labeled processes in the 315 ventromedial PFC, specifically infralimbic and prelimbic areas $(96.2 \%$ reduction, 316 paired t-test: WT vs. dKO: $P=0.003)$ with fibers present in the anterior white 317 matter for both (Fig. 4e; Extended Data Fig. 6d). Because there is reduced RA 318 activity in the outer shell of the striatum in the dKO brain (Extended Data Fig. 3a), we 319 assessed the trajectories of axon fibers through the striatum. We found that fibers 320 transit through the medial aspect of the striatum (Extended Data Fig. 6c,d), suggesting 
321 that alterations in RA signaling in the striatum do not affect guidance of reciprocal 322 mPFC-MD connectivity. In addition, reduction of connections between the mPFC and

323 thalamus was not due to cell death in the mPFC (Paired t-test: WT vs. dKO: $P=0.618$; 324 Extended Data Fig. 7e).

325 While we saw no changes in RA activity in the dKO thalamus (Extended Data

326 Fig. 3a), RA signaling has been previously implicated in thalamic development ${ }^{53}$, thus

327 we assessed whether other thalamocortical connections were altered in the dKO mice

328 at P5 using DTI. There was no difference in thalamocortical connectivity with the MOp, 329 primary auditory area (AUDp), or SSp (Paired $t$-test: WT vs. dKO: $\mathrm{P}=0.7,0.6,0.3$ for 330 MOp, AUDp, and SSp, respectively) (Fig. 4a,c; Extended Data Fig. 7b). Consistent 331 with this, formation of barrel fields in the SSp in P5 control and dKO brains showed no 332 significant difference (Extended Data Fig. 7c). Given that Aldh1a3 is expressed in the $333 \mathrm{ACA}^{51}$, we also examined whether thalamocortical innervation was altered in the ACA, 334 but found no difference in the number of streamlines (Paired t-test: WT vs. dKO: $P=$ 335 0.3) (Fig. 4a; Extended Data Fig. 7b). The corticospinal tract (CST) and connections 336 across the corpus callosum between left and right mPFC, SSp and MOp in the dKO 337 showed no difference in the number of streamlines compared to control (Paired t-test: 338 WT vs. dKO: $\mathrm{P}=0.5,0.4,0.5,0.12$ for CST, mPFC-mPFC, SSp-SSp, and MOp-MOp, 339 respectively: Fig. 4a,c, Extended Data Fig. 7b). Furthermore, scalar indexes, which 340 describe the microstructural integrity of white matter, were similar between WT and dKO 341 mice in the corpus callosum, anterior commissure and internal capsule (Extended Data

342 Fig. 7a). In addition, the width of the CST at P30 was slightly increased in the dKO mice 343 (Two-tailed Student's t-test: WT vs. dKO: $\mathrm{P}=0.03$; Extended Data Fig. 7d). In 344 summary, deletion of Rxrg and Rarb leads to reduction of RA signaling specifically in 345 mPFC, as well as selective reduction of reciprocal mPFC-MD connectivity.

347 Ectopic retinoic acid signaling expands medial thalamic connectivity and 348 promotes layer 4 molecular identity

349 In primates, MD innervation of the frontal cortex is expanded laterally compared to 350 rodents, with the primate PFC areas have a more prominent layer 4 16,18,21. Thus, we 351 investigated whether expansion of RA signaling is sufficient to increase and laterally 352 extend MD innervation and alter layer 4 development in the mouse neonatal frontal 
353 cortex by genetically deleting the RA-degrading enzyme CYP26B1 in mice (Extended

354 Data Fig. 8a). Whereas heterozygotes appear normal and are fertile, mice with

355 constitutive deletion of Cyp26b1 exhibited various body malformations and died

356 perinatally. Nevertheless, it has been previously shown that the genetic deletion of

357 Cyp26b1 in mice resulted in expansion of RA signaling in multiple organs prenatally ${ }^{54}$

358 and the postnatal mouse mPFC ${ }^{38}$. Consistent with previous findings in humans ${ }^{32}$ and

359 mice ${ }^{38}$, CYP26B1 is upregulated in M1C compared to the PFC during human mid-fetal

360 development (Fig. 1g) and upregulated in the anterior insula and MOs/MOp of the

361 neonatal mouse frontal neocortex (Fig. 5a; Extended Data Fig. 8b). To investigate the

362 possible role of CYP26B1 in restricting RA signaling to the mouse medial frontal cortex,

363 we generated Cyp26b1 KO mice that also harbored the RARE-lacZ transgene. The lack

364 of Cyp26b1 resulted in spreading of RA signaling dorso-laterally toward the MOs and

365 MOp regions of the RARE-lacZ reporter mouse line at 18 PCD (Two-tailed Student's t-

366 test: WT vs. KO: $\mathrm{P}=0.004,0.04$ for $\mathrm{mPFC}$ in section 1 and 2, respectively; $\mathrm{P}=0.006$,

3670.04 for MOs in section 1 and 2, respectively; $P=0.01,0.0001$ for MOp in section 1 and

368 2, respectively; Fig. 5b; Extended Data Fig. 8a). This increase was not robustly 369 observed in more posterior regions (Extended Data Fig. 8c).

370 Next, we used Cyp26b1 KO mice to study the effects of expansion of RA 371 signaling into the dorso-lateral frontal cortex, by placing Dil into the medial thalamus of 372 fixed postmortem WT and KO brains, harvested at E18 due to perinatal lethality of the $373 \mathrm{KO}$ mice. Histological analysis of the E18 brains revealed that WT littermates had 374 occasional thalamocortical axons within the medial and dorso-lateral frontal white 375 matter and cortex at this age. In contrast, KO mice showed precocious and robust 376 innervation of both the medial and dorso-lateral frontal cortex by the axons originating 377 from the medial thalamus (657\% increase; Two-tailed Student's t-test: WT vs. KO: $P=$ 378 0.0004; Fig. 5c, Extended Data Fig. 9b). We also observed moderately enlarged 379 frontal cortex (Two-tailed Student's t-test: WT vs. KO, P = 0.0001 for frontal cortex size) 380 and grossly typical cytoarchitecture of the cortical wall and cortical plate analyzed areas 381 of the Cyp26b1 KO cortex (Extended Data Fig. 10a, b).

$382 \quad$ Furthermore, we observed an upregulation and expansion in laminar expression 383 of layer 4 marker, retinoic acid-related orphan receptor beta (Rorb), in the frontal cortex 384 of Cyp26b1 KO mice (Two-tailed Student's t-test: WT vs. KO, $\mathrm{P}=0.0002$ in bin 2; $\mathrm{P}=$ 
3850.01 in bin 3; Fig. 5d; Extended Data Fig. 9a). Similarly, misexpression of a plasmid 386 expressing Aldh1a3, in the dorso-lateral fronto-parietal cortex using in utero 387 electroporation lead to the expansion of Rorb expression domain and ectopic 388 expression of Rorb in deep layers at P0 (Two-tailed Student's t-test: control vs. Aldh1a3389 electroporated: $P=0.0001$; Extended Data Fig. 10c).In summary, we identified that 390 ectopic RA signaling in the perinatal mouse frontal cortex leads to expansion of 391 thalamocortical innervation as well as regional and laminar expansion of expression of 392 layer 4 marker, Rorb, , both characteristics of the lateral granular PFC in anthropoid 393 primates $^{16}$.

\section{Conclusion}

396 In this study, we report that spatial regulation of RA concentration and signaling during 397 the mid-fetal period is important for PFC development. We identified a high anterior to 398 low posterior gradient of RA in the developing primate cortex and of RA receptors, Rxrg 399 and Rarb, in the developing neocortex of both rodents and primates. The graded 400 expressions of transcription factors, PAX6 and NR2F1/COUP-TF1, predominantly in 401 early cortical progenitor cells, are required for early regional patterning of the cerebral 402 cortex $^{11,13-15,55,56}$. It is conceivable that the anterior-posterior gradient of RA is refined 403 by interplay with the PAX6 anterior-posterior gradient and an opposing NR2F1 404 posterior-anterior gradient. Pax6 expression can be induced by RA $^{57}$ and PAX6 405 positively regulates Aldh1a3 expression ${ }^{50}$, whereas NR2F1 has been shown to repress 406 RA-stimulated transcription 57. Furthermore, in the developing retina, RA 407 regulates FGF8 ${ }^{58}$ which was previously shown to regulate the patterning of the frontal 408 cortex in mice ${ }^{10,12}$. Thus, graded RA signaling may directly interact with previously 409 identified cortical patterning signaling mechanisms.

410 We showed that Rxrg and Rarb are required for proper development of mPFC-MD 411 long-range connectivity in mouse. Interestingly, there is a ventro-lateral and dorso412 lateral expansion of $A L D H 1 A 3$ expression in the mid-fetal primate neocortex, similar to 413 the expansion of PFC-MD connectivity in primates, and a posterior shift of Cyp26b1 414 expression, co-incident with posterior expansion of the primate PFC. Long-range 415 connectivity with the thalamus has previously been shown to be integral for specification 416 of the primary and secondary sensory cortex ${ }^{11}$, and was recently reported to regulate 
417 Cyp26b1 in the mouse frontal cortex ${ }^{38}$. Of note, mid-fetal cortical development in human 418 and macaque, and equivalent perinatal/neonatal development in mice, is a critical 419 period for laminar and areal specification of neurons, onset of dendritic growth and 420 synaptogenesis, axon pathfinding, and ingression of thalamocortical axons ${ }^{28}$. Taking 421 this evidence together, we propose expansion of RA signaling is a mechanism for 422 expansion of association areas in the primate PFC through the expansion of innervation 423 from the MD nucleus of the thalamus. Noteworthy, while our study has not revealed 424 how changes in RA signaling may regulate thalamocortical innervation, we did observe 425 several genes encoding axon guidance molecules that are enriched in the neonatal 426 frontal cortex and dysregulated in mice lacking Rxrg and Rarb. Furthermore, we also 427 found that an increase in RA signaling in the mouse neocortex leads to the expansion 428 of Rorb expression, a key marker of granular layer 4, which is a distinct feature of the laterally expanded primate PFC $16,18,20,21$.

430 The PFC is hypothesized to have been derived from two prime entities: the ventral 431 paleocortical moiety that evolved from the olfactory/pyriform system and the dorsal 432 archicortical moiety that evolved from the dorsal hippocampus ${ }^{58}$. Interestingly, we 433 identified the mid-fetal frontal and temporal lobe, which are adjacent to each moiety, as 434 having the most differentially expressed genes during mid-fetal neocortical 435 development. Noteworthy, RA signaling is involved in the development of both 436 entitites ${ }^{36,37}$. In mice, the meninges covering the hippocampus, which resides next to 437 the temporal neocortex, express the other two RA synthesizing genes (Aldh1a1/Raldh1 438 and Aldh1a2/Raldh2) and RA signaling plays a critical role in hippocampal 439 neurogenesis and function ${ }^{33}$. RA signaling is also required for the development of the 440 olfactory system and its long-range connections with the ventrolateral forebrain ${ }^{37}$. 441 Interestingly, we also identified an enrichment of RA in the ITC of humans, but not 442 macaques. The ITC is an association area in the temporal lobe involved in object and 443 face recognition and exhibits unique specialization and connectivity in humans ${ }^{23,49}$. 444 Noteworthy, the expression of the serum retinol-binding protein gene is progressively 445 restricted to the higher-order fronto-parieto-temporal association areas during the 446 postnatal development of macaque neocortex ${ }^{59}$. As expansion of the prefrontal and 447 temporal association areas have been proposed to be one of the evolutionary 448 underpinnings of advanced cognition $17,19,20,22,23$, it will be important to explore whether 
449 RA signaling has a broader role in developmental specification and expansion of 450 association areas.

451 Clinically, disruptions in PFC development and reciprocal long-range 452 connections between the MD nucleus of the thalamus and the PFC are thought to 453 underlie cognitive dysfunction in schizophrenia ${ }^{9}$ and pathophysiology of ASD $^{5}$. 454 Disruption of RA signaling has been implicated in schizophrenia ${ }^{45-48}$, including 455 enrichment of rare variation mutations in $R A R B$ in cases with severe cognitive deficits ${ }^{48}$. 456 Furthermore, RA is known teratogen that can cause defects in human cerebral cortical 457 development ${ }^{60}$. Our findings identify a possible mechanistic link between RA 458 dysregulation, cognitive dysfunction, and neuropsychiatric and neurodevelopmental 459 disorders.

460 


\section{References}

482 1. Goldman-Rakic P.S. (1987) Circuitry of primate prefrontal cortex and regulation 483 of behavior by representational memory. Handb. Physiol. 5, 373-417

484 2. Miller, E. K. \& Cohen, J. D. An Integrative Theory of Prefrontal Cortex Function. $485 \quad$ Annu. Rev. Neurosci. 24, 167-202 (2001).

486 3. Arnsten, A. F. T. Stress signalling pathways that impair prefrontal cortex structure and function. Nat. Rev. Neurosci. 10, 410-422 (2009).

488 4. Fuster, J. M. The Prefrontal Cortex (Elsevier, 2015).

489 5. Amaral, D. G., Schumann, C. M. \& Nordahl, C. W. Neuroanatomy of autism. Trends Neurosci. 31, 137-45 (2008).

6. Willsey, A. J. et al. Coexpression networks implicate human midfetal deep cortical projection neurons in the pathogenesis of autism. Cell 155, 997-1007 (2013).

7. Tan, H.-Y., Callicott, J. H. \& Weinberger, D. R. Dysfunctional and compensatory prefrontal cortical systems, genes and the pathogenesis of schizophrenia. Cereb. Cortex 17, i171-81 (2007).

8. Gulsuner, S. et al. Spatial and temporal mapping of de novo mutations in schizophrenia to a fetal prefrontal cortical network. Cell 154, 518-529 (2013).

9. Giraldo-Chica, M., Rogers, B. P., Damon, S. M., Landman, B. A. \& Woodward, N. D. Prefrontal-thalamic anatomical connectivity and executive cognitive function in schizophrenia. Biol. Psychiatry 83, 509-517 (2018).

10. Fukuchi-Shimogori, T. \& Grove, E. A. Neocortex patterning by the secreted signaling molecule FGF8. Science 294, 1071-1074 (2001).

11. Sur, M. \& Rubenstein, J. L. R. Patterning and plasticity of the cerebral cortex. Science 310, 805-810 (2005).

12. Cholfin, J. A. \& Rubenstein, J. L. R. Frontal cortex subdivision patterning is coordinately regulated by Fgf8, Fgf17, and Emx2. J. Comp. Neurol. 509, 144155 (2008).

13. O'Leary, D. D. \& Sahara, S. Genetic regulation of arealization of the neocortex. Curr. Opin. Neurobiol. 18, 90-100 (2008).

14. Geschwind, D. H. \& Rakic, P. Cortical evolution: Judge the brain by its cover. Neuron 80, 633-647 (2013). 
513 15. Cadwell, C. R., Bhaduri, A., Mostajo-Radji, M. A., Keefe, M. G. \& Nowakowski,

514 T. J. Development and arealization of the cerebral cortex. Neuron 103, 980$5151004(2019)$.

516 16. Preuss, T. M. Do rats have prefrontal cortex? The rose-woolsey-akert program 517 reconsidered. J. Cogn. Neurosci. 7, 1-24 (1995).

518 17. Semendeferi, K., Lu, A., Schenker, N. \& Damasio, H. Humans and great apes share a large frontal cortex. Nat. Neurosci. 5, 272-6 (2002).

18. Uylings, H. B. M., Groenewegen, H. J. \& Kolb, B. Do rats have a prefrontal cortex? Behav. Brain Res. 146, 3-17 (2003).

19. Schoenemann, P. T., Sheehan, M. J. \& Glotzer, L. D. Prefrontal white matter volume is disproportionately larger in humans than in other primates. Nat. Neurosci. 8, 242-52 (2005).

20. Petrides, M., Tomaiuolo, F., Yeterian, E. H. \& Pandya, D. N. The prefrontal cortex: comparative architectonic organization in the human and the macaque monkey brains. Cortex. 48, 46-57 (2012).

21. Carlén, M. What constitutes the prefrontal cortex? Science 358, 478-482 (2017).

22. Smaers, J. B., Gómez-Robles, A., Parks, A. N. \& Sherwood, C. C. Exceptional evolutionary expansion of prefrontal cortex in great apes and humans. Curr. Biol. 27, 1549 (2017).

23. Donahue, C. J., Glasser, M. F., Preuss, T. M., Rilling, J. K. \& Van Essen, D. C. Quantitative assessment of prefrontal cortex in humans relative to nonhuman primates. Proc. Natl. Acad. Sci. U. S. A. 115, 5183-5192 (2018).

24. Maden, M. Retinoic acid in the development, regeneration and maintenance of the nervous system. Nat. Rev. Neurosci. 8, 755-65 (2007).

25. Rhinn, M. \& Dollé, P. Retinoic acid signalling during development. Development

539 26. Pletikos, M. et al. Temporal specification and bilaterality of human neocortical topographic gene expression. Neuron 81, 321-32 (2014).

54127 Li, M. et al. Integrative functional genomic analysis of human brain development and neuropsychiatric risks. Science 80. 362, (2018). 
543 28. Silbereis, J. C., Pochareddy, S., Zhu, Y., Li, M. \& Sestan, N. The cellular and molecular landscapes of the developing human central nervous system. Neuron 89, 248-68 (2016).

546 29. Chiang, M. Y. et al. An essential role for retinoid receptors RAR $\beta$ and RXRY in 547 long-term potentiation and depression. Neuron 21, 1353-1361 (1998).

548 30. Krezel, W. et al. Impaired locomotion and dopamine signaling in retinoid receptor mutant mice. Science 279, 863-867 (1998).

550 31. Shibata, M., Pattabiraman, K., Muchnik, S.K., and Sestan, N. Hominini-specific regulation of CBLN2 increases prefrontal synaptogenesis. Submitted.

32. Johnson, M. B. et al. Functional and evolutionary insights into human brain development through global transcriptome analysis. Neuron 62, 494-509 (2009).

33. McCaffery, P., Zhang, J. \& Crandall, J. E. Retinoic acid signaling and function in the adult hippocampus. J. Neurobiol. 66, 780-91 (2006).

34. Wagner, E., Luo, T., Sakai, Y., Parada, L. F. \& Dräger, U. C. Retinoic acid delineates the topography of neuronal plasticity in postnatal cerebral cortex. Eur. J. Neurosci. 24, 329-340 (2006).

35. Chen, N. \& Napoli, J. L. All-trans-retinoic acid stimulates translation and induces spine formation in hippocampal neurons through a membrane-associated RARalpha. FASEB J. 22, 236-45 (2008).

36. Siegenthaler, J. A. et al. Retinoic acid from the meninges regulates cortical neuron generation. Cell 139, 597-609 (2009).

37. Choi, J., Park, S. \& Sockanathan, S. Activated retinoid receptors are required for the migration and fate maintenance of subsets of cortical neurons. Dev. 141, $1151-1160$ (2014).

38. Larsen, R., Proue, A., Scott, E. P., Christiansen, M. \& Nakagawa, Y. The thalamus regulates retinoic acid signaling and development of parvalbumin interneurons in postnatal mouse prefrontal cortex. eNeuro 6, (2019). differentiation prefigure olfactory pathway formation in the mammalian forebrain. Neuron 10, 1035-1048 (1993). 
574 40. Dmetrichuk, J. M., Carlone, R. L. \& Spencer, G. E. Retinoic acid induces neurite outgrowth and growth cone turning in invertebrate neurons. Dev. Biol. 294, 3949 (2006).

41. Rothwell, C. M., de Hoog, E. \& Spencer, G. E. The role of retinoic acid in the formation and modulation of invertebrate central synapses. J. Neurophysiol. 117, 692-704 (2017).

42. Moreno-Ramos, O. A., Olivares, A. M., Haider, N. B., de Autismo, L. C. \& Lattig, M. C. Whole-exome sequencing in a south american cohort links ALDH1A3, FOXN1 and retinoic acid regulation pathways to autism spectrum disorders. PLoS One 10, e0135927 (2015).

43. Xu, X. et al. Excessive UBE3A dosage impairs retinoic acid signaling and synaptic plasticity in autism spectrum disorders. Cell Res. 28, 48-68 (2018).

44. Kumar, S. et al. Impaired neurodevelopmental pathways in autism spectrum disorder: a review of signaling mechanisms and crosstalk. J. Neurodev. Disord. 11, 10 (2019).

45. Goodman, A. B. Three independent lines of evidence suggest retinoids as causal to schizophrenia. Proc. Natl. Acad. Sci. U. S. A. 95, 7240-4 (1998).

46. Lamantia, A. S. Forebrain induction, retinoic acid, and vulnerability to schizophrenia: insights from molecular and genetic analysis in developing mice. Biol. Psychiatry 46, 19-30 (1999).

47. Lerner, V., McCaffery, P. J. A. \& Ritsner, M. S. Targeting retinoid receptors to treat schizophrenia: Rationale and progress to date. CNS Drugs 30, 269-80 (2016).

50. Suzuki, R. et al. Identification of RALDH-3, a novel retinaldehyde dehydrogenase, expressed in the ventral region of the retina. Mech. Dev. 98,

604 51. Smith, D., Wagner, E., Koul, O., McCaffery, P. \& Dräger, U. C. Retinoic acid 605 synthesis for the developing telencephalon. Cereb. Cortex 11, 894-905 (2001). 
606 52. Rossant, J., Zirngibl, R., Cado, D., Shago, M. \& Giguere, V. Expression of a retinoic acid response element-hsplacZ transgene defines specific domains of transcriptional activity during mouse embryogenesis. Genes Dev. 5, 1333-1344 (1991).

53. Wilde, J. J., Siegenthaler, J. A., Dent, S. Y. R. \& Niswander, L. A. Diencephalic size is restricted by a novel interplay between GCN5 acetyltransferase activity and retinoic acid signaling. J. Neurosci. 37, 2565-2579 (2017).

54. Yashiro, K. et al. Regulation of retinoic acid distribution is required for proximodistal patterning and outgrowth of the developing mouse limb. Dev. Cell 6, 411-422 (2004).

55. Armentano, M. et al. COUP-TFI regulates the balance of cortical patterning between frontal/motor and sensory areas. Nat. Neurosci. 10, 1277-86 (2007).

56. Qiu, Y., Krishnan, V., Pereira, F. A., Tsai, S. Y. \& Tsai, M. J. Chicken ovalbumin upstream promoter-transcription factors and their regulation. J. Steroid Biochem. Mol. Biol. 56, 81-5 (1996).

57. Gajovic, S., St-Onge, L., Yokota, Y. \& Gruss, P. Retinoic acid mediates Pax6 expression during in vitro differentiation of embryonic stem cells. Differentiation 62, 187-92 (1997).

58. da Silva, S. \& Cepko, C. L. Fgf8 expression and degradation of retinoic acid are required for patterning a high-acuity area in the retina. Dev. Cell 42, 68-81 (2017).

59. Komatsu, Y., Watakabe, A., Hashikawa, T., Tochitani, S. \& Yamamori, T. Retinol-binding protein gene is highly expressed in higher-order association areas of the primate neocortex. Cereb. Cortex 15, 96-108 (2005). 


\section{Methods}

639 Human developmental brain RNA-seq analysis. Bulk-tissue human brain 640 developmental RNA-seq data (counts file) with its metadata information was 641 downloaded from development.psychencode.org. A total of 73 mRNA samples 642 corresponding to 11 possible neocortical areas/regions from windows 3 and 4 (16-22 643 PCW) were considered for analyses (Extended Data Fig. 1). The human neocortical 644 areas under study are orbital (oPFC/OFC), dorso-lateral (dIPFC/DFC), ventro-lateral 645 (vIPFC/VFC), medial (mPFC/MFC) prefrontal cortex, and primary motor cortex (M1C) 646 from the frontal lobe; primary somatosensory cortex (S1C) and posterior inferior parietal 647 cortex (IPC) from the parietal lobe; primary auditory cortex (A1C), posterior superior 648 temporal cortex (STC), and inferior temporal cortex (ITC) from the temporal lobe; and 649 primary visual cortex (V1C) from the occipital lobe. A TMM normalization procedure was 650 applied (function normalizeCounts from tweeDEseq package in $\mathrm{R}$ ) to the expression of 651 15,724 protein-coding genes that show sufficiently large counts (determined with 652 function filterByExpr from edgeR package in R). To identify genes that are upregulated 653 in a given brain lobe, we first applied RNentropy ${ }^{61}$ available as a package in $\mathrm{R}$, to 654 determine which genes are differentially expressed among the 11 neocortical areas. 655 Then, we considered a gene to be specifically overexpressed in a given lobe if i) there 656 is at least one area in this lobe where the gene is significantly upregulated, ii) the gene 657 is not upregulated in any area of the other lobes, and iii) the gene is under-expressed 658 in at least $30 \%$ of the areas from the remaining lobes. Similarly, we identified genes that 659 are specifically upregulated in the PFC compared to the M1C, or vice versa, by first 660 running RNentropy pairwise comparisons between M1C and each of the prefrontal 661 areas independently. Then, a gene was considered to be upregulated in PFC if i) it was 662 upregulated in a prefrontal area in at least one of the comparisons, ii) it was not 663 upregulated in M1C in any of the comparisons, and iii) it was under-expressed in M1C 664 in at least three of the comparisons. A gene was considered to be upregulated in M1C 665 if i) it was overexpressed in MOp in at least three of the comparisons, ii) it was not 666 upregulated in any PFC area, and iii) it was under-expressed in a prefrontal area in at 667 least one of the comparisons. Principal component analyses were performed using the 668 prcomp function in $\mathrm{R}$ by centering the log2-transformed expression data of the selected 669 genes. Significant GO terms were obtained via goana function from the limma package 
670 in $\mathrm{R}$, reporting the ones with at least 10 genes in the background and at least 5 in the

671 dataset. Sequencing data were deposited at http://psychencode.org and NCBI dbGAP

672 Accession phs000755.v2.p1

673

674 Animals. All experiments using animals were performed in accordance with

675 protocols approved by the Yale University Institutional Animal Care and Use

676 Committee (IACUC). The day on which a vaginal plug was observed was designated 677 as 0.5 post conception day (PCD). RARE-lacZ (Tg(RARE-Hspa1b/lacZ)12Jrt) mice,

678 and Timed pregnant CD-1 mice for in utero electroporation were purchased from

679 Jackson Laboratory and Charles River Laboratories, respectively.

680

681

682

Generation of Rxrg, Rarb, and Cyp26b1 knockout mice using CRISP-Cas9 gene

683 editing technique. The overall strategy for the generation of Rxrg and Rarb KO mice follows a previously described protocol using CRISPR-Cas9 genome editing technique ${ }^{62}$. For the construction of the templates of guidance RNA, two sets of top and bottom strand oligomers (see Supplementary Table 4) directing the double strand break at targeting sites were annealed and ligated into $\mathrm{Bbs} /$ site of $\mathrm{pX} 330$ vector $^{63}$. After amplification of insert with T7-tagged primers (see Supplementary Table 5), guidance RNAs were synthesized by T7 RNA polymerase. The coding 690 the pSP64 Poly(A) vector (Promega). Vectors were digested and linearized with 691 EcoRI. Caspase mRNA was synthesized by SP6 RNA polymerase. Guidance RNAs 692 and Cas9 mRNA were purified by MEGAclear Transcription Clean-Up Kit (Ambion).

693 Cas9 mRNA, and two guidance RNAs were mixed at a concentration of (10 ng; 100 $\left.694 \mathrm{ng} ; 100 \mathrm{ng} \mathrm{H}^{-1}\right)$ in the microinjection buffer (5mMTris-HCl pH7.5; 0.1M EDTA) and 695 injected into the pronuclei of fertilized eggs from B6SJLF1/J mouse strain. The first 696 generation (F0) mice with recombined alleles were identified by PCR with two primer 697 sets designed outside and inside of targeted area (Supplementary Table 5; 698 Extended Data Fig. 2d,9a), confirmed by sequencing. The germ line transmission 699 in F1 generation was confirmed by the same sets of PCR primers. For generation of 700 Rxrg KO mice, a pair of guidance RNAs flanking whole exon 3 and 4 were designed 701 to delete a large part of DNA binding domain (Extended Data Fig. 2d) ${ }^{29}$. For 
702 generation of Rarb KO mice, a pair of guidance RNAs were designed to delete the

703 whole of exon 9 and a part of exon 10 (Extended Data Fig. 2d). As a result, $\alpha$ -

704 helical sheets of $\mathrm{H} 4$ to $\mathrm{H} 8$ in the ligand binding domain were deleted and a frame

705 shift occurred in the rest of C-terminal region, which results in total abolition of

706 receptor activity ${ }^{64}$. For generation of Cyp26b1 KO mice, a pair of guidance RNAs

707 were designed to delete the whole of exon 3 and 6 as described previously to abolish

708 enzymatic activity (Extended Data Fig. 8a) ${ }^{65}$. All primer sequences are listed in

709 Supplementary Table 5.

710

711 In situ hybridization. Section in situ hybridization was performed as described

712 previously ${ }^{66}$. Antisense digoxigenin (DIG)-labeled RNA probes were synthesized

713 using DIG RNA Labeling Mix (Roche). Human and mouse ALDH1A3 (Clone ID

714 6208628, and 6515355, respectively), RXRG (Clone ID 4635470, and 5707723,

715 respectively), $R A R B$ (Clone ID 30341884, and 30608242, respectively), and mouse

716 Rorb (Clone ID 5358124), Cbln2 (Clone ID 6412317), Cyp26b1 (Clone ID 6400154)

717 cDNAs were purchased from GE Healthcare. Mouse Meis2 DNA was a gift from

718 John Rubenstein. For macaque in situ hybridization, human probes were used

719 because of high similarity between human and macaque transcripts $(95.4 \%$ identity

720 in $A L D H 1 A 3 ; 97.7 \%$ in RXRG; 98.8\% in RARB). Sections were obtained from 21, 22

721 PCW human brains and 110, 114 PCD macaque brains. In situ hybridization were

722 repeated using these 2 sets. Rorb intensity was quantified using Image J. Rorb

723 thickness was quantified by dividing the cortical plate into 5 equal bins and intensity

724 in each bin was quantified using Aperio ImageScope (Leica).

725

726 Enzyme-linked immunosorbent assay (ELISA). Eleven neocortical areas were

727 dissected from four fresh frozen postmortem human mid-fetal brains $(16,18,18$ and 19

728 PCW) and four fresh frozen macaque brains (all four 110 PCD) as described in Zhu et

729 al. $2018^{67}$. Each sample was further microdissected into three pieces and weighed.

730 Each piece was independently homogenized using a dounce homogenizer in three to

731 four volumes of homogenizing buffer (isopropanol: ethanol=2:1; $1 \mathrm{mg} / \mathrm{ml}$ butylated

732 hydroxytoluene), followed by centrifugation at $10 \mathrm{~K} \mathrm{rpm}$ for 10 minutes at $4{ }^{\circ} \mathrm{C}$.

733 Supernatant was used for both determination of protein concentration by BCA kit 
734 (Thermo-Fisher Scientific), and RA concentrations using ELISA colorimetric detection

735 Kit according to the manufacturer's instructions (Cat. MBS705877, MyBioSource, San

736 Diego, CA). This kit could not distinguish forms (all trans retinol, all trans retinal, and all

737 trans retinoic acid). Thus, the concentration of RA was the overall concentration.

739 Quantitative reverse transcription-PCR. Total RNA was isolated from freshly 740 microdissected cortices after removal of the olfactory bulb and striatum using Trizol 741 (Thermo-Fisher Scientific). cDNAs were prepared using SuperScript II (Invitrogen) from 742 three independent WT cerebral hemispheres. Quantitative reverse transcription (RT)-

743 PCR was performed as described previously ${ }^{68}$. At least three replicates per transcript 744 were used for every reaction. The copy number of transcripts was normalized against 745 the house keeping TATA-binding protein (TBP) transcript level. For Rxra,b,g, Rara,b,c 746 and Tbp primer sets, correlation (R2) was higher than 0.98, and the slope was -3.1 to $747-3.6$ in each standard curve. Primers to detect the expression of the genes above were 748 designed in a single exon. Primer sequences are listed in Supplementary Table 5.

$750 \quad \beta$-Galactosidase histochemical staining. Brains were dissected from P0 RARE751 lacZ mouse pups and drop-fixed in 4\% paraformaldehyde for 2 hours at 4C, followed 752 by embedding in OCT. Brains were sectioned at $20 \mu \mathrm{m}$ by cryostat (Leica CM3050S) 753 after they were frozen. $\beta$-Galactosidase staining followed the protocol described by 754 Kokubu et $a^{69}$. We used Red-gal (Sigma-Aldrich) for the chromogenic reaction.

755 Intensity of $\beta$-Galactosidase staining was quantified using Image J.

757 Nissl staining. Postmortem brains were harvested and fixed with $4 \%$ 758 paraformaldehyde overnight at $4^{\circ} \mathrm{C}$, followed by embedding in OCT. Brains were 759 sectioned at $15-20 \mu \mathrm{m}$ by cryostat (Leica CM3050S) after they were frozen. After 760 PBS wash, sections were dehydrated using increasing concentration of ethanol, 761 followed by cresyl violet, wash, and second ethanol dehydration.

763 Immunohistochemistry. The brains were dissected from each embryo and fixed 764 with $4 \%$ paraformaldehyde overnight at $4{ }^{\circ} \mathrm{C}$, followed by embedding in OCT. Brains 765 were sectioned at $15-20 \mu \mathrm{m}$ by cryostat (Leica CM3050S) after they were frozen. 
766 The sources of primary antibodies were anti-L1CAM (1:500; Millipore), anti-cleaved

767 capase3 (1:500; Cell Signaling), anti-PSD95/DLG4 (1:500; Invitrogen). Secondary

768 antibodies: Alexa Fluor 488- or 594- conjugated AffiniPure Donkey anti-Rabbit IgG

769 (Jackson ImmunoResearch). For all microscopic analysis, LSM510 META (Zeiss),

770 and LSM software ZEN were used.

771

772 Quantification of postsynaptic puncta marked by PSD-95/DLG4

773 immunostaining. For each region of both WT and dKO mice, using the $488 \mathrm{~nm}$

774 channel to detect DLG4/PSD-95 and DAPI to label nuclei, seven serial optical

775 sections at $0.8 \mu \mathrm{m}$ intervals over a total depth of $5 \mu \mathrm{m}$ were imaged and the $2^{\text {nd }}, 4^{\text {th }}$,

776 and $6^{\text {th }}$ images were eliminated from further analysis to avoid overlap in counting ${ }^{70}$.

777 Area of each image is $0.079 \mathrm{~mm}^{2}$. The number of PSD-95 puncta on each image

778 was counted automatically using Image J using threshold of 985 to 4095 and analyze

779 particles function. At least two sections from each animal were selected for counting,

780 and at least 3 animals for each genotype were used.

782 Anterograde tracing of axons. For anterograde tracing of axons between mPFC 783 and thalamus, brains were collected at either 18 PCD or P21, and fixed overnight in $7844 \%$ paraformaldehyde at $4{ }^{\circ} \mathrm{C}$. Brains were then hemidissected. A crystal of $1,1 \#-$ 785 dioctadecyl-3,3,3\#,3\# tetra-methyl-indocarbocyanine perchlorate (Dil, Sigma786 Aldrich) was inserted either into the mPFC, MD nucleus of the thalamus, or medial 787 thalamus under the stereomicroscope. The size of the crystal is $\sim 200 \mu \mathrm{m}$. Brains 788 were then placed in $1 \%$ paraformaldehyde in PBS and left for 14 days at $37{ }^{\circ} \mathrm{C}$. 789 Following Dil diffusion, the brains were sectioned coronally on a vibrating microtome 790 (Leica) at $80 \mu \mathrm{m}$ thickness and stained with DAPI. Sections were mounted onto 791 glass and immediately sealed in VECTASHIELD Hardset Antifade Mounting Medium 792 (VECTOR Laboratories). Slides were analyzed under ApoTome.2 microscope 793 (Zeiss) and intensity was quantified using ImageJ.

794 
795 Plasmid construction. For construction of expression vectors, full-length cDNAs

796 (mouse Aldh1a3, Clone ID 6515355, purchased from GE Healthcare) were inserted

797 into pCAGIG vector (pCAGIG was obtained from Addgene (Plasmid \# 11159).

798

799 In utero electroporation. In utero electroporation was performed as previously 800 described ${ }^{68}$. Plasmid DNA $(4 \mu \mathrm{g} / \mu \mathrm{l})$ was injected into the lateral ventricle of 801 embryonic mice at E13.5-E14.5 and transferred into the cells of the ventricular zone 802 by electroporation (five 50 -ms pulses of $40 \mathrm{~V}$ at $950-\mathrm{ms}$ intervals). Brains were 803 dissected at P0. Brains and tissue sections of electroporated animals were analyzed

804 for GFP expression after fixation with 4\% paraformaldehyde at P0.

806 Mouse RNA-seq data generation and analysis. Mouse brains were dissected at 807 P0 in ice-cold sterile PBS, fresh frozen, and stored at $-80^{\circ} \mathrm{C}$. Brains were incubated 808 in RNAlater-Ice at $-20^{\circ} \mathrm{C}$ for $12-16$ hours prior to further dissection. mPFC, MOs, and 809 OFC were microdissected based on Paxinos and Frankin, $2007^{71}$ and the Allen 810 Mouse Brain Atlas (mouse.brain-map.org/static/atlas) ${ }^{72}$ and RNA was isolated using 811 RNeasy Plus Micro kit with additional on-column DNAase step (Qiagen). RNA quality 812 and amount were quantified using High Sensitivity RNA Screen Tape assay (Agilent), 813 and concentration was standardized to $10 \mathrm{ng} / \mathrm{ul}$. SMART-seq v4 Ultra Low Input Kit 814 (Takara) was used to create cDNA, and concentration was quantified using Quant815 iT Picogreen kit (Thermo-Fisher Scientific). Nextera XT DNA library Prep Kit 816 (Illumina) were used to create cDNA libraries for sequencing. Libraries were 817 normalized and sequenced at the Yale Center for Genomic Analysis (YGCA) using 818 the NovaSeq with 100 bp paired end reads. Reads from each library were mapped 819 against the mouse assembly GRCm38 using STAR v.2.6.0a (gtf and fasta files 820 downloaded from Ensembl version 94; parameters: --readFilesIn \$j1 \$j2 -821 outSAMattributes All --outFilterMultimapNmax 1 --outSAMstrandField intronMotif -822 outFilterIntronMotifs RemoveNoncanonical --quantMode TranscriptomeSAM -823 outFilterMismatchNoverLmax 0.1 --alignSJoverhangMin 8 --alignSJDBoverhangMin 8241 --outSAMunmapped Within --outFilterType BySJout). Counts were obtained using 825 featureCounts v1.6.2 with -p parameter. 
To compare the gene expression patterns of three WT vs three Rarb/Rxrg dKO mice, a TMM a procedure was applied (function normalizeCounts from tweeDEseq package in R) to the expression of 15085 protein-coding genes that show sufficiently large counts (determined with function filterByExpr from edgeR 830 package in R). We assessed DEx genes in each brain region (mPFC, OFC, and 831 MOs) running RNentropy independently among WT and dKO mice per region. Genes 832 overexpressed in a given condition are those that are both significantly upregulated 833 in that condition and significantly downregulated in the opposite condition according 834 to RNentropy. The same criterion was applied for identification of downregulated 835 genes. Genes with an inconsistent pattern of expression between regions were 836 excluded. Principal component analyses were performed using the prcomp function 837 in $\mathrm{R}$ by centering the log2-transformed expression data of the selected genes. 838 Significant GO terms were obtained via goana function from the limma package in $R$ 839 and plotted using function GOBubble from GOplot package in R. Fisher test 840 enrichments calculated for RA related genes (RA synthesis: Rdh10, Rdh5, Raldh1, 841 Raldh2, Raldh3, Adh1, Adh5, Adh7; RA degrading: Cyp26a1, Cyp26b1, Cyp26c1:

842 RA receptor: Rara, Rarb, Rarg, Rxra, Rxrb, Rxrg; RA binding: Ttr, Rlbp1, Rbp1, $843 R b p 2, R b p 3, R b p 4$, Fabp5), genes overexpressed in individual lobes of the mid-fetal 844 human cortex based on Fig. 1, and neuropsychiatric disease related genes 845 (downloaded from Li et al. ${ }^{27}$ ) in up- and downregulated genes. Genes associated 846 with the GO terms: "axon guidance”, "axon guidance receptor", “axon development”, 847 and "ephrin" were manually screened for anterior to posterior gradient using 848 developingmouse.brain-map.org ${ }^{73}$, gensat.org ${ }^{74}$ and Elsen et al. ${ }^{75}$

850 Retinoic acid receptor binding site analysis. We identified all those protein coding 851 genes associated to GO terms related to axon guidance and development, and synapse 852 formation among those genes upregulated in the mid-fetal human frontal cortex or up853 and down-regulated in the Rxrg and Rarb dKO mice, whose human orthologs were 854 identified using Ensemble Biomart ( 430 genes). This produced six lists of genes of 855 which we then collected H3K27ac peaks active in mid-fetal human dIPFC/DFC from a 856 collapsed PsychENCODE Chip-Seq dataset reported in Li et al., $2018^{27}$ containing 857 dorsal frontal cortex and cerebellum samples from mid-fetal and adult brains. From the 
858 hg38 coordinates of each of the $\sim 1700$ peaks obtained, we downloaded their DNA 859 sequence from the UCSC Genome Browser using twoBitToFa ${ }^{76}$. We then run FIMO 860 with default parameters to predict TFBS in those sequences using 8 JASPAR motifs 861 associated to retinoic acid receptors in meme format ${ }^{77}$. For each gene and motif, we 862 counted how many matches we observed in its putative cis-regulatory elements (CRE). 863 We divided the number of bases covered by a given motif by twice the total length of 864 each gene CREs, because our search space was in both the forward and reverse 865 strand. We then discretized the proportion of sites spanning putative RARB/RXRG 866 binding sites in five quantiles by including the union of the gene lists and calculated the 867 proportion of genes falling in the five quantiles, plus a category representing genes with 868 no predicted binding sites, and a category representing genes with no identified CREs. 869 To compare these proportions with a null expectation, we performed randomization 870 among the $\sim 15,000$ protein coding genes sufficiently expressed in human frontal cortex 871 and used in the differential expression analysis. The randomization strategy consisted 872 of i) sampling 100 times a number of genes equal to the union of the six gene lists and,

873 ii) for each of those random groups, sample a number of genes equal to the size of each 874 individual list. This strategy could better approximate the notable gene overlap observed 875 among those gene sets. Finally, we classified each gene from the randomized groups 876 in the same quantiles categories derived from the union of the six gene lists. For each 877 motif, we analyzed which gene lists presented categories with proportions falling outside 878 the $95 \%$ confidence interval of the 100 randomizations. We tested for enrichment of 879 RARB/RXRG binding sites around genes (-500 bp/+100 bp and +/- 10,000 bp of the 880 transcriptional start site (TSS)) using RcisTarget ${ }^{78}$, and in the H3K27ac peaks from the 881 previous section with HOMER findMotifsGenome.pl ${ }^{79}$.

883 Diffusion-weighted magnetic resonance imaging and tractography. Following 884 proper euthanasia, five dKO homozygotes and five WT C57BL/6 mice at P5 were 885 sacrificed and brains were dissected. Brains were drop fixed in a paraformaldehyde 886 solution of $4 \%$ in $0.1 \mathrm{M}$ phosphate buffered saline (PBS) for 48 hours. They were 887 subsequently transferred to $0.1 \mathrm{M}$ PBS and just before imaging to Fomblin (Sigma888 Aldrich St Louis, MO, USA). The diffusion-weighted images were acquired on a Bruker 889 BioSpin 9.4 T MRI (Bruker GmbH, Ettlingen, Germany) using a standard 3D Stejskal- 
890 Tanner spin-echo sequence with 30 different angles of diffusion sensitization at $b$ value

891 of $1000 \mathrm{~s} / \mathrm{mm}^{2}$ and the following parameters: repetition time $=2000 \mathrm{~ms}$; echo 892 time $=25.616 \mathrm{~ms}$; diffusion encoding duration $=4 \mathrm{~ms}$. The in-plane resolution was

$8930.11 \mathrm{~mm}$ and slice thickness was $0.22 \mathrm{~mm}$. Overall scanning time was $\sim 24$ hours.

895 Image processing and tractography. Cerebral cortical regions of interest (ROI) 896 and thalamus were manually defined according to Paxinos and Frankin ${ }^{71}$ and the 897 Allen Mouse Brain Atlas (mouse.brain-map.org/static/atlas) by D.A. and K.P. without 898 prior knowledge of the experimental groups. The reconstruction of axonal pathways 899 was performed with MRtrix $3^{80}$ software using constrained spherical deconvolution ${ }^{81}$ 900 and probabilistic tracking (iFOD2) with FOD amplitude cut-off of 0.1 . The thalamus 901 was used as a seeding point and each cortical ROI was used as a termination mask. 902 To evaluate the integrity of the major white matter tracts between the groups, both 903 internal capsules, anterior commissure and corpus callosum were manually 904 delineated according to Paxinos and Frankin, $2007^{71}$ and the Allen Mouse Brain 905 Atlas (mouse.brain-map.org/static/atlas) ${ }^{72}$ by D.A. and K.P. without prior knowledge 906 of the experimental groups. Values of the fractional anisotropy (FA), apparent 907 diffusion coefficient (ADC), radial (RD) and axial (AD) diffusivity were calculated 908 using underlying scalar maps derived by MRtrix3.

910 Processing, analysis, and image visualization. To allow robust visualization and 911 analysis, images depicting Dil tracing or immunohistochemistry using antibody 912 against PSD95 have been inverted and/or pseudo colored, as in Fig. 4, 5. 


\section{Extended References}

923 61. Zambelli, F. et al. RNentropy: An entropy-based tool for the detection of significant variation of gene expression across multiple RNA-Seq experiments. Nucleic Acids Res. 46, (2018).

62. Wang, $\mathrm{H}$. et al. One-step generation of mice carrying mutations in multiple genes by CRISPR/cas-mediated genome engineering. Cell 153, 910-918 (2013).

63. Cong, L. et al. Multiplex genome engineering using CRISPR/Cas systems. Science 339, 819-823 (2013).

64. Renaud, J. P. et al. Crystal structure of the RAR-y ligand-binding domain bound to all-trans retinoic acid. Nature 378, 681-689 (1995).

65. Maclean, G., Li, H., Metzger, D., Chambon, P. \& Petkovich, M. Apoptotic extinction of germ cells in testes of Cyp26b1 knockout mice. Endocrinology 148, 4560-4567 (2007).

66. Wilkinson, D. G. \& Nieto, M. A. Detection of messenger RNA by in situ hybridization to tissue sections and whole mounts. Methods Enzymol. 225, 36173 (1993).

68. Shibata, M., Kurokawa, D., Nakao, H., Ohmura, T. \& Aizawa, S. MicroRNA-9 modulates Cajal-Retzius cell differentiation by suppressing Foxg1 expression in mouse medial pallium. J. Neurosci. 28, 10415-10421 (2008).

69. Kokubu, H. \& Lim, J. X-gal staining on adult mouse brain sections. Bio Protoc. 4, pii: e1064 (2014).

70. Ippolito, D. M. \& Eroglu, C. Quantifying synapses: An immunocytochemistrybased assay to quantify synapse number. J. Vis. Exp. 45, pii: 2270 (2010).

71. Paxinos, G. Atlas of the Developing Mouse Brain: at E17.5, PO, and P6. (Academic Press, 2007).

951 73. Thompson, C. L. et al. A high-resolution spatiotemporal atlas of gene expression 952 of the developing mouse brain. Neuron 83, 309-323 (2014). 
953 74. Heintz, N. Gene expression nervous system atlas (GENSAT). Nat. Neurosci. 7, $954 \quad 483(2004)$

955 75. Elsen, G. E. et al. The protomap is propagated to cortical plate neurons through 956 an Eomes-dependent intermediate map. Proc. Natl. Acad. Sci. U. S. A. 110, 957 4081-4086 (2013).

958 76. W. J. Kent et al., The human genome browser at UCSC. Genome Res. 12, 996$9591006(2002)$.

960 77. A. Mathelier et al., JASPAR 2016: a major expansion and update of the open961 access database of transcription factor binding profiles. Nucleic Acids Res. 44, 962 D110-D115 (2016).

963 78. S. Aibar et al., SCENIC: single-cell regulatory network inference and clustering. Nat. Methods. 14, 1083-1086 (2017)

79. S. Heinz et al., Simple combinations of lineage-determining transcription factors prime cis-regulatory elements required for macrophage and B cell identities. Mol. Cell. 38, 576-589 (2010)

80. Tournier, J.-D., Calamante, F. \& Connelly, A. MRtrix: Diffusion tractography in crossing fiber regions. Int. J. Imaging Syst. Technol. 22, 53-66 (2012).

81. Tournier, J. D., Calamante, F. \& Connelly, A. Robust determination of the fibre orientation distribution in diffusion MRI: Non-negativity constrained superresolved spherical deconvolution. Neuroimage 35, 1459-1472 (2007). 


\section{Acknowledgements}

986 We thank Suxia Bai and Timothy Nottoli for technical help in generation of gene-edited

987 mouse lines; Fahmeed Hyder and Jelena Mihailovic for their assistance with

988 MRI diffusion-weighted sequence design and conducting the MRIs; Alvaro Duque for

989 use of equipment from MacBrainResource (MH113257); John Rubenstein for sharing

990 reagents; and the members of Sestan laboratory for comments. This work was

991 supported by the National Institutes of Health (MH106874, MH106934, MH110926,

992 MH116488), and Simons Foundation (N.S.). The project that gave rise to these results

993 received the support of a fellowship from "la Caixa" Foundation (ID 100010434). The

994 fellowship code is LCF/BQ/PI19/11690010. Additional support was provided by the

995 Kavli Foundation and the James S. McDonnell Foundation (N.S.).

996

\section{Author Contributions}

998 M.S., K.P., and N.S. designed the research; M.S. and K.P. performed mouse experiments, 999 analyzed the data; B.L.G. analyzed human and mouse transcriptomic datasets; G.S. 1000 analyzed the enrichment of binding sites for RA receptors; M.S. generated construct for 1001 mutant mice lines; X.X., performed pronuclear injection; D.A. analyzed mouse imaging 1002 data; A.M.M.S. dissected postmortem human and macaque tissues used for analysis; 1003 N.S. conceived the study; M.S., K.P., and N.S. wrote the manuscript. All authors 1004 discussed the results and implications and commented on the manuscript at all stages.

1007 The authors declare no competing financial interests. 
a Mid-fetal human brain
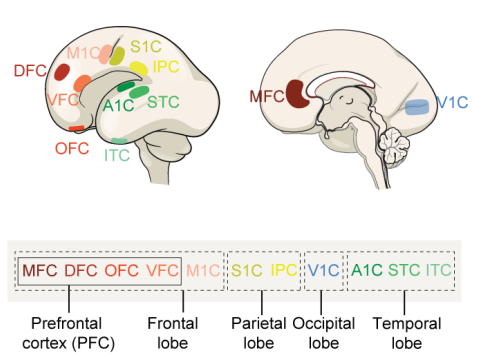

d

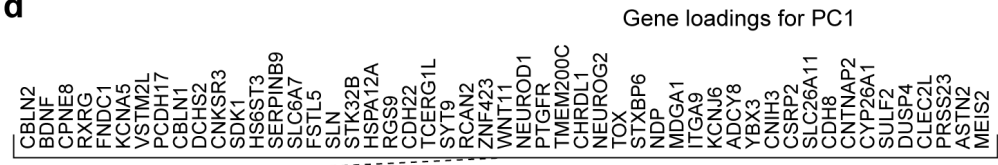

b PCA (11 neocortical areas)

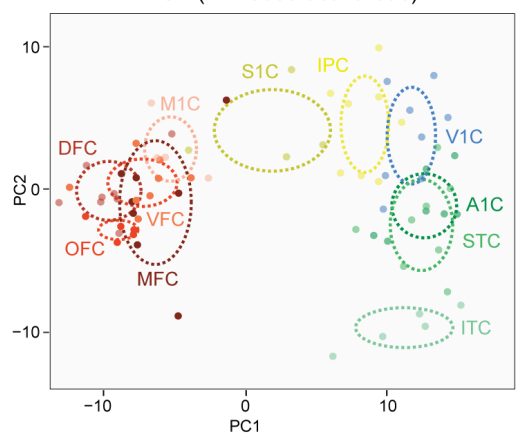

C GO terms (Frontal lobe genes)

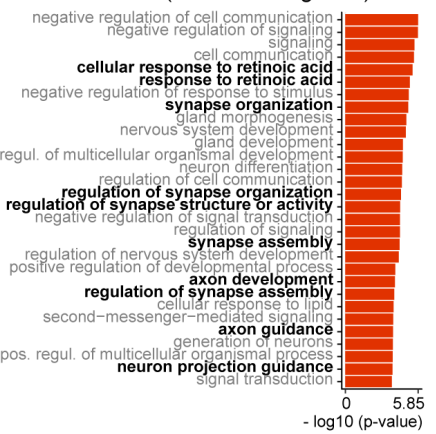

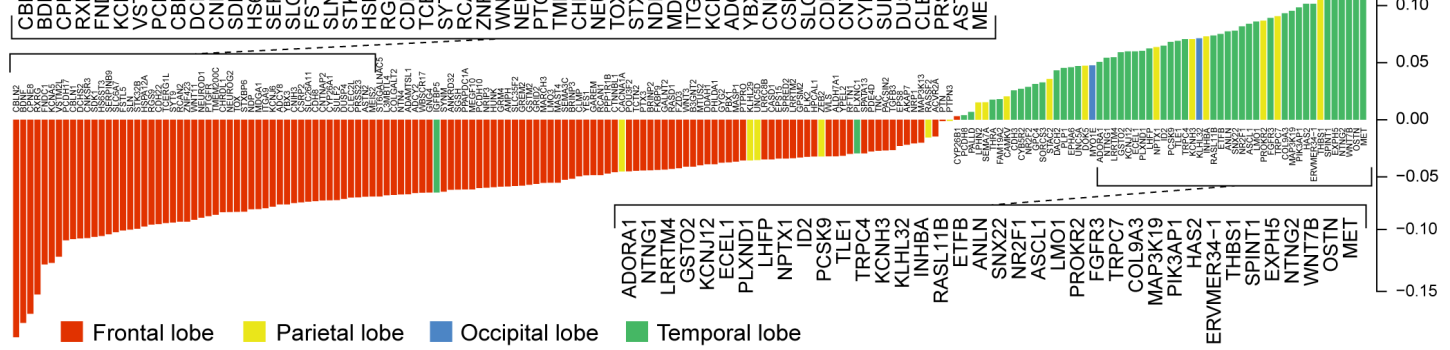

e

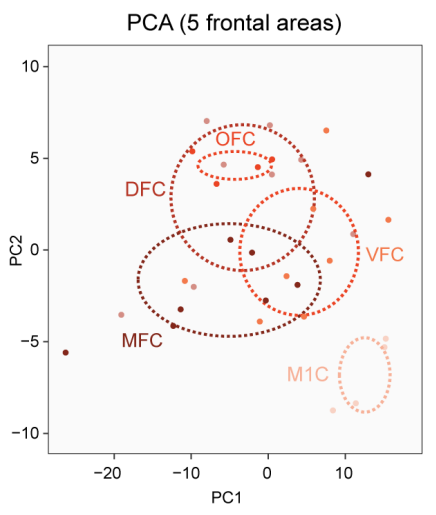

f

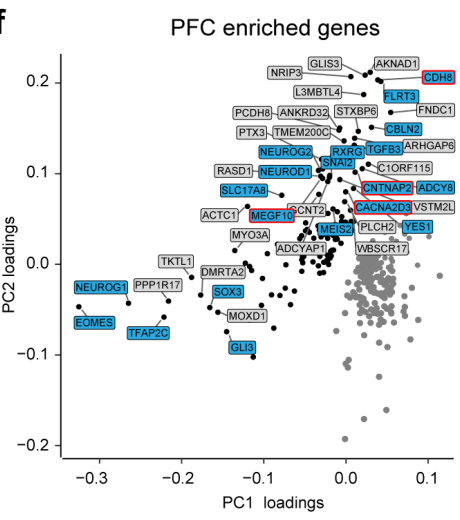

M1C enriched genes

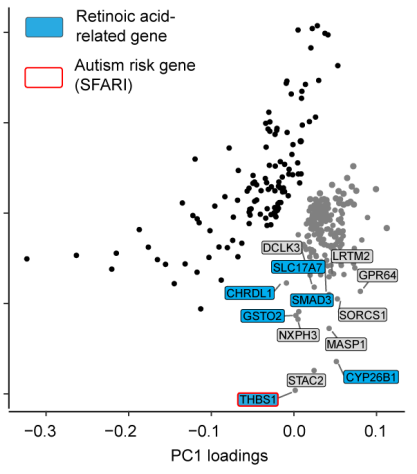

Figure 1. Retinoic acid-related genes are upregulated in human mid-fetal PFC. a, Diagram of the 11 human mid-fetal neocortical areas from the four brain lobes used for analysis. b. First two principal components based on the expression of 190 protein-coding genes that are specifically upregulated in one of the four brain lobes. Each color represents a neocortical area. Ellipses are centered on the mean of the points of a given area and the size of the axes corresponds to their standard deviation on each component. c, GO terms associated with the genes specifically upregulated in the frontal lobe and their nominal P value. d, Gene loadings of the first principal component. Colors represent the brain lobe where the gene was found to be specifically upregulated. e, First two principal components based on the expression of 306 genes differentially expressed between PFC and M1C. f, Loadings of the first two principal components with labeled genes being upregulated in PFC (black dots) and M1C (gray dots). Genes highlighted in blue are associated with RA signaling, and genes with red outline have ASD risk alleles. 


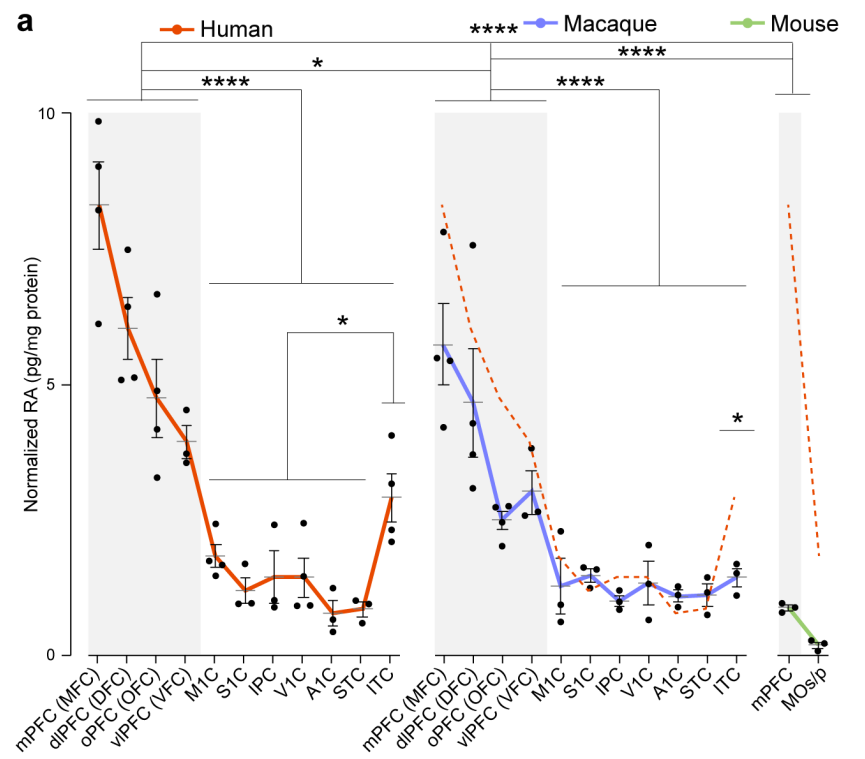

b $\quad$ Human $\quad$ Macaque $\quad$ Mouse

C
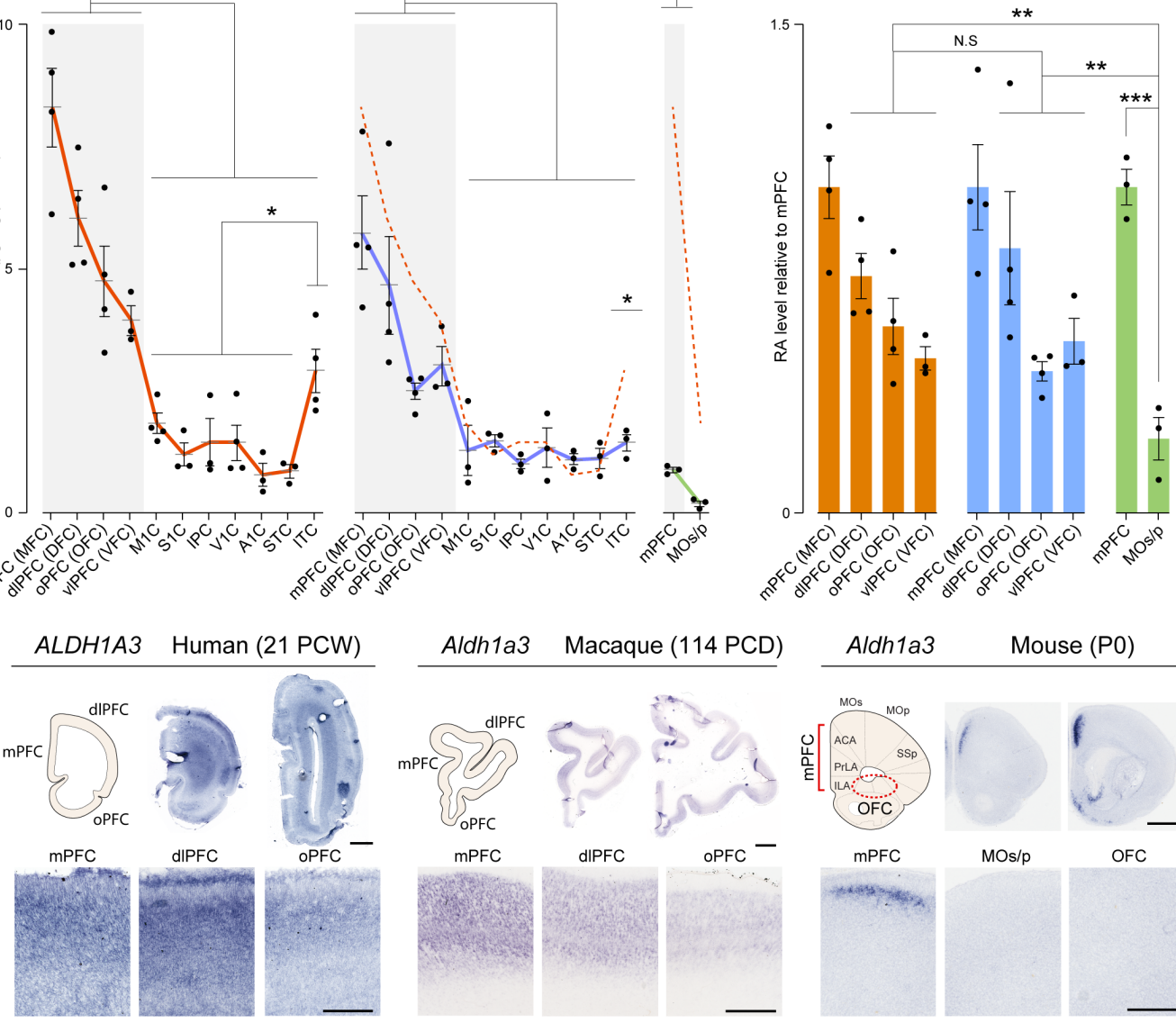

Figure 2. Lateral expansion of the retinoic acid synthesizing enzyme, ALDH1A3 in primate mid-fetal frontal lobe. a, Quantification of RA concentration by ELISA from 11 cortical areas from all four neocortical lobes of the human (16, 18, 19, 21 PCW) and macaque (Four 110 PCD brains), and mPFC and MOs from mouse (Four P1 brains) (N = 3-4 for each areal sample). Details about dissection of postmortem brain subregion samples are described in Methods. RA amount was normalized by the total protein in the lysate. Two-tailed unpaired t-test: ${ }^{* * * *} \mathrm{P}<5 \mathrm{e}-5$, ${ }^{*} \mathrm{P}<0.05 ; \mathrm{N}=3-4$ per condition; Errors bars: S.E.M. Dashed red line in macaque and mouse graphs represent human RA concentration. b, Relative RA level in MPFC, dIPFC, vIPFC compared to mPFC (human, macaque) or mPFC, MOs compared to MPFC (mouse). Two-tailed ratio paired t-test: ${ }^{* * *} \mathrm{P}=0.0004,{ }^{* *} \mathrm{P}<0.005, \mathrm{~N}=3-4$; Errors bars: S.E.M. c, Schema of the PFC and representative images of cortical ALDH1A3 expression in coronal sections of human (21 PCW), macaque (114 PCD), and mouse (P0) brains using in situ hybridization. Expression in human and macaque MPFC, dIPFC, and oPFC, and mouse mPFC comprised of infralimbic (ILA), prelimbic (PrLA), and anterior cingulate area (ACA), MOs/p, and OFC are shown at higher magnification. Note that ALDH1A3/Aldh1a3 expression is expanded to all regions of the anterior part of the midfetal human and macaque frontal cortex (PFC), while it is restricted to the medial frontal 1050 cortex (mPFC) in mouse. Scale bars: $200 \mu \mathrm{m}$ (mouse); $2 \mathrm{~mm}$ (human and macaque); $100 \mu \mathrm{m}$ (mouse, lower panel); $500 \mu \mathrm{m}$ (human and macaque, lower panel). $\mathrm{N}=2$ for human and macaque; $\mathrm{N}=3$ for mouse. 

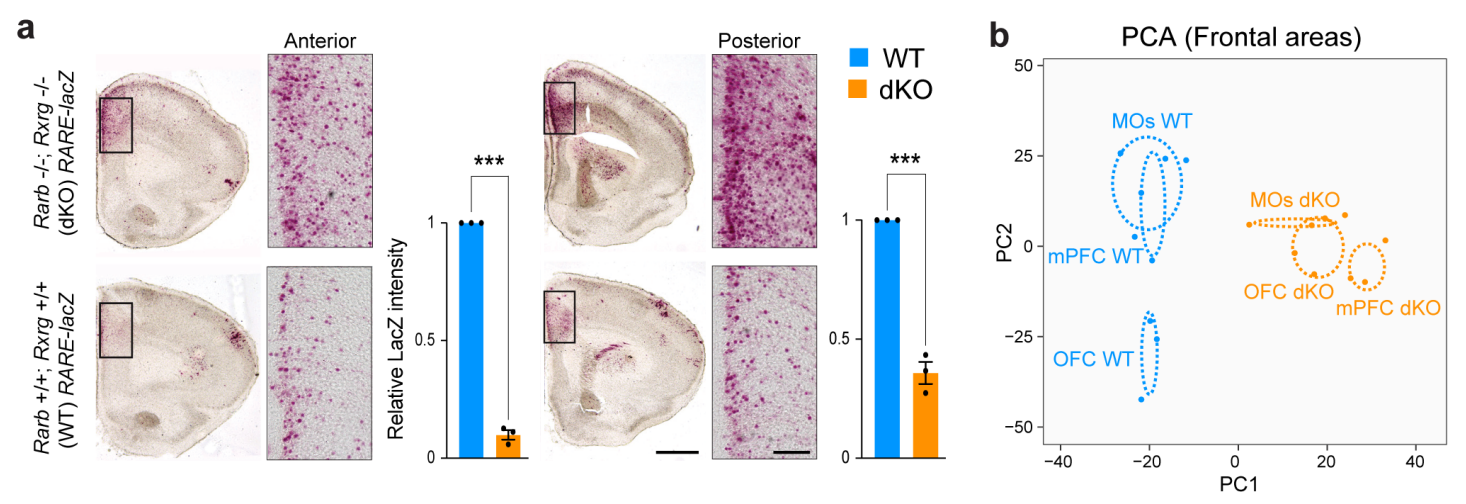

C

GO terms: Biological process

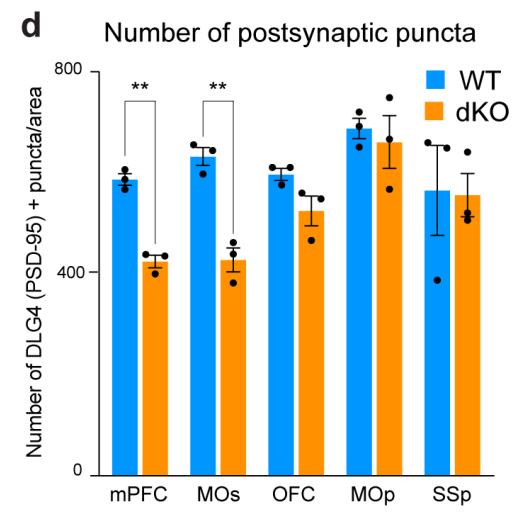

Figure 3. Reduced RA signaling in the PFC of mice lacking RXRG and RARB leads to downregulation of genes involved in synapse and axon development. $a, \beta$ Galactosidase histochemical staining in the mouse PFC from Rxrg+/+; Rarb+/+; (WT); RARE-lacZ (Blue) and Rxrg-l-; Rarb-/- (dKO); RARE-lacZ (orange) brains at PO. In the $\mathrm{dKO}$ brains, LacZ signal is reduced in the MPFC (inset) ( $\mathrm{N}=3$ per genotype). Twotailed Student's t-test: WT vs. dKO: ${ }^{* \star *} P<0.0005$; Errors bars: S.E.M.; Scale bars: 200 $\mu \mathrm{m} ; 50 \mu \mathrm{m}$ (inset). b, First two principal components calculated from the expression of 4,768 protein-coding genes that are differentially expressed between WT and dKO mice in at least one of the three frontal cortex areas (mPFC, MOs, and OFC). c, GO terms associated with the 4,768 DEx genes showing their Z-score and nominal $P$ values. Zscore represents the proportion of upregulated versus downregulated genes in the dKO compared to WT in the list of DEx genes associated to each GO term (i.e. $z$-score $=$ (\#up - \#down) / sqrt (\#all DEx associated to the GO term)). Dark blue: z-score <-5; light blue: $z$-score $(-5,0]$; orange: $z$-score $>0$. Size of the bubbles are proportional to the total number of DEx genes associated to the given GO term. $\mathbf{d}$, Quantification of excitatory synapses marked by PSD-95/DLG4 in the MPFC, MOs, OFC, MOp, and SSp regions from PO WT and dKO mice brains. Two-tailed Student's t-test: ${ }^{* *} P<0.005$; Errors bars; 

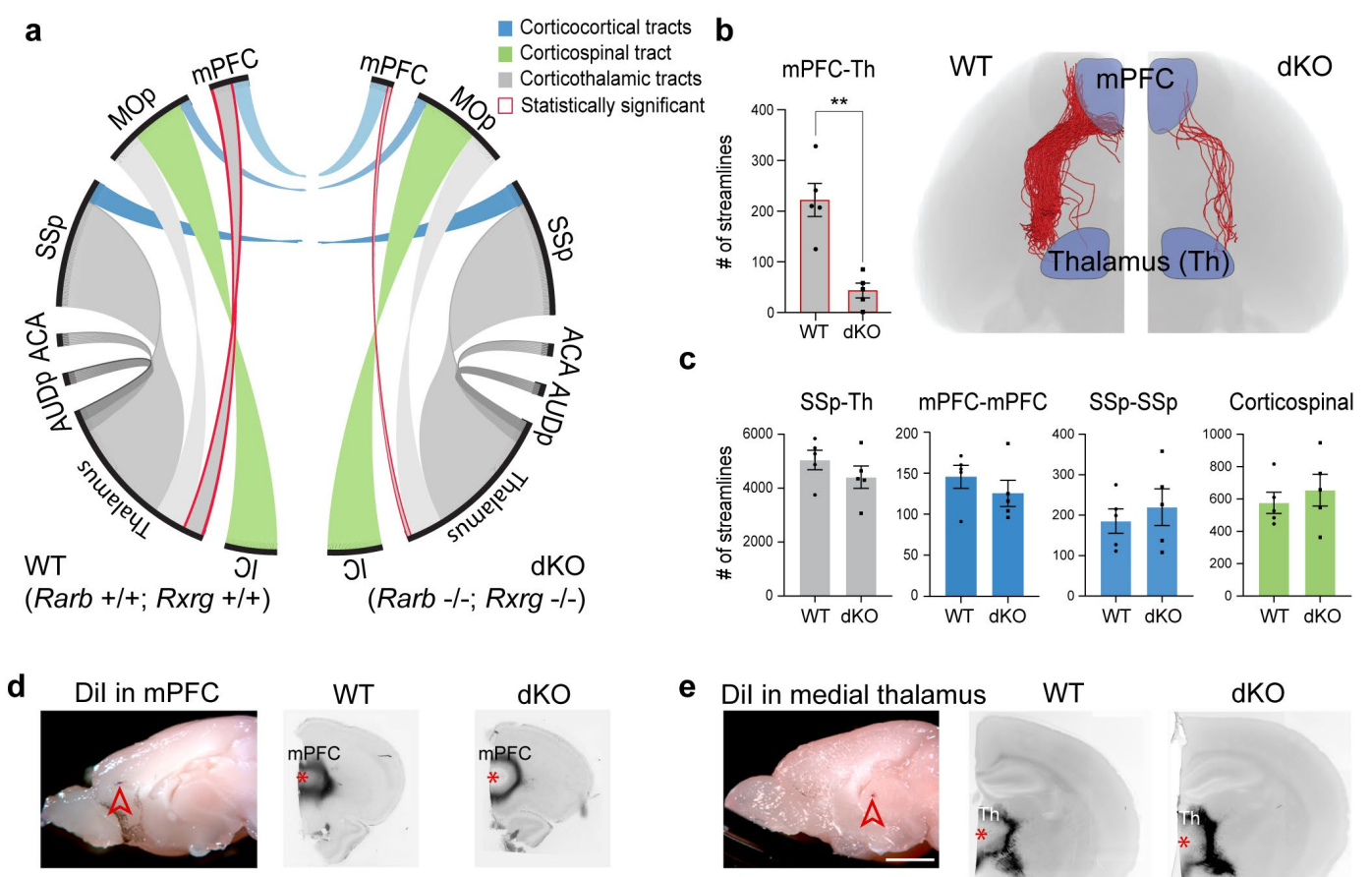

e Dili
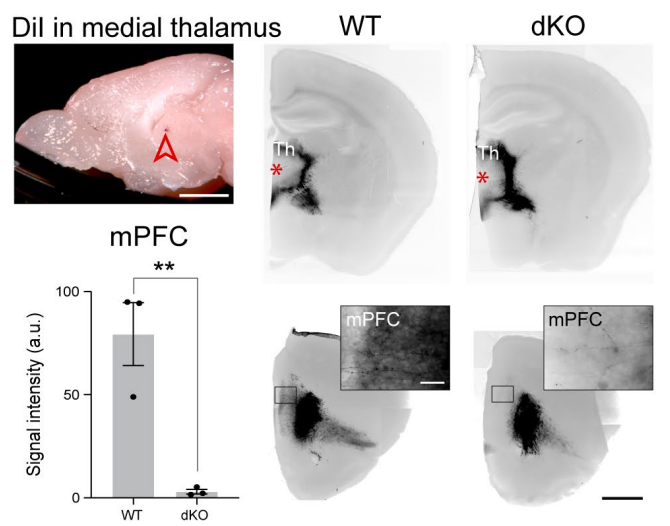

Figure 4. Loss of mPFC-MD thalamic connections in mice lacking Rarb and Rxrg. a, Representation of number of streamlines (NOS) generated as a connectivity measurement between the cortical areas, thalamus and internal capsule (IC) at P5 using DTI. In dKO brains, there is a significant decrease in NOS generated between the thalamus and MPFC. NOS between other regions are not significantly different between $\mathrm{dKO}$ and WT brains ( $\mathrm{N}=5$ per genotype). $\mathbf{b}$, Visualization and quantification of streamlines between mPFC and thalamus in dKO and the WT brains. Paired t-test: ${ }^{* *} P$ $<0.005 ; \mathrm{N}=5$ per genotype. c, Quantification of select corticothalamic, corticocortical and corticospinal streamlines. Paired t-test: NS; Errors bars: S.E.M; $N=5$. d, e, Dil placement in the mPFC and medial thalamus in WT and dKO brain at P21. In dKO brains, the labelled processes detected in the mediodorsal (MD) thalamus (inset), and mPFC (inset) are significantly reduced, indicating the reduction of reciprocal thalamocortical and corticothalamic projections. Two-tailed Student's t-test: ${ }^{*} \mathrm{P}=0.009$, ${ }^{* *} \mathrm{P}=0.003 ; \mathrm{N}=3$ per genotype; Errors bars: S.E.M.; Scale bars: $1 \mathrm{~mm} ; 100 \mu \mathrm{m}$ (inset). 
a

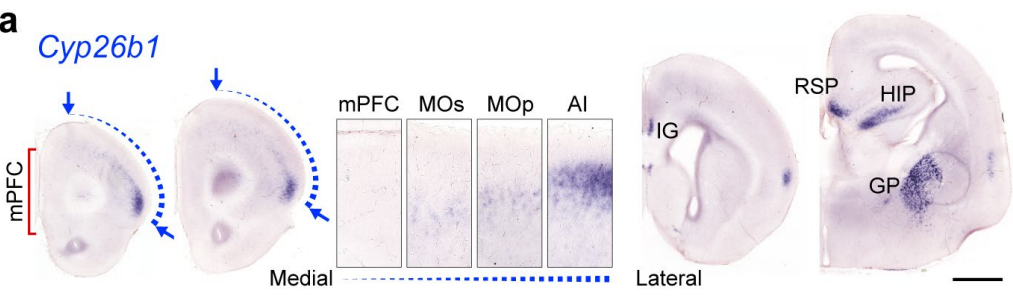

b

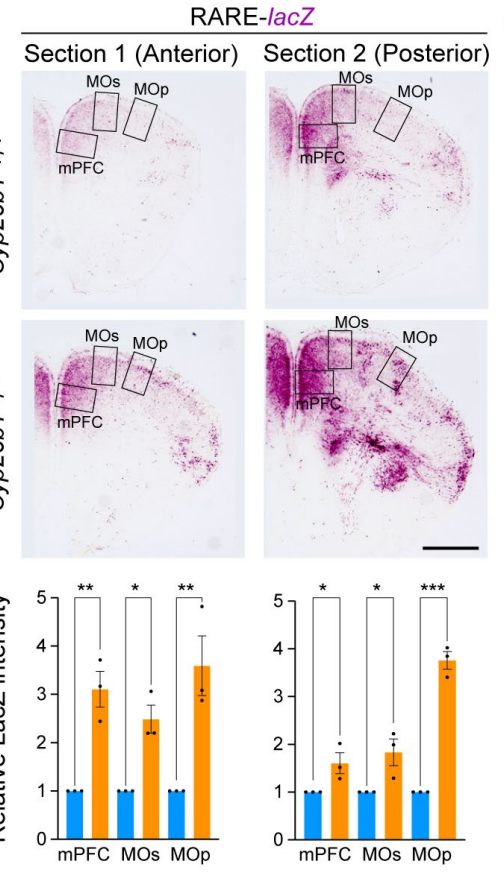

C

d Rorb

Dil in medial thalamus
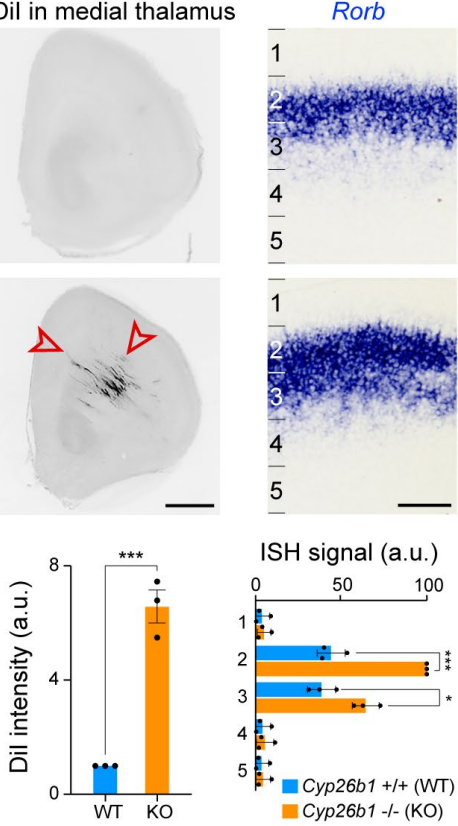

ISH signal (a.u.)

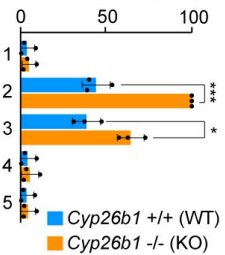

Figure 5. Increased retinoic acid signaling promotes mPFC-medial thalamic connectivity. a, Expression of Cyp26b1 in the P0 mouse brain using in situ hybridization. Cyp26b1 expression displays a gradient from the insula (Al) to MOs (insets). Arrows indicate Cyp26b1 expression at the insula and MOs. Scale bars, 200 $\mu \mathrm{m} . \mathrm{N}=4 \mathbf{b}, \beta$-Galactosidase histochemical staining of the mPFC of Cyp26b1+/+ (WT); RARE-lacZ and Cyp26b1-/- (KO); RARE-lacZ mouse brains at 18 PCD. Significant increase in signal intensity was observed in Cyp26b1 KO brains in the mPFC, MOs, and MOp. Signal intensity was quantified in the boxed area (mPFC, MOs, and MOp). Twotailed Student's t-test: WT vs. Cyp26b1 KO: ${ }^{* *} \mathrm{P}<0.0005,{ }^{* *} \mathrm{P}<0.005$, ${ }^{*} \mathrm{P}<0.05$; $\mathrm{N}=$ 3 per genotype; Errors bars: S.E.M. c, Dil labeling in 18 PCD frontal cortex after Dil was placed in the MD thalamus of WT and Cyp26b1 KO brains. A significant increase in the density of labeled ingressing thalamocortical axons was observed in the perinatal Cyp26b1 KO frontal cortex. Two-tailed Student's t-test: ${ }^{* * *} \mathrm{P}=0.0004 ; \mathrm{N}=3$ per genotype and condition. Scale bars: $200 \mu \mathrm{m}$. d, The expression of Rorb, a gene expressed preferentially in layer 4 neurons, is upregulated in Cyp26b1 KO mice. The cortex was divided into five equal regions spanning from the pia to the ventricular zone, and signal intensity in each region is quantified for each genotype. Two-tailed Student's t-test: WT vs. Cyp26b1 KO: ${ }^{* * *} \mathrm{P}=0.0002,{ }^{*} \mathrm{P}=0.01 ; \mathrm{N}=3$ per genotype; Errors bars: S.E.M. IG, Indusium griseum; GP, Globus pallidus; HIP, Hippocampus; RSP, Retrosplenial cortex. 
$\mathbf{a}_{\text {Age }}$
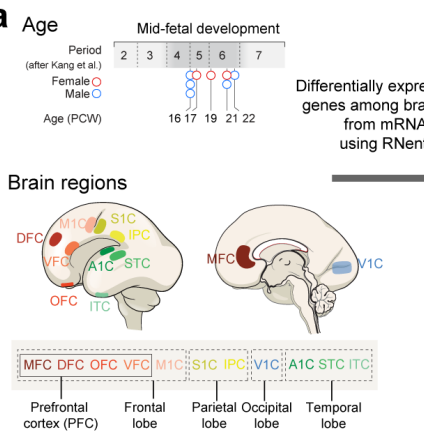

b

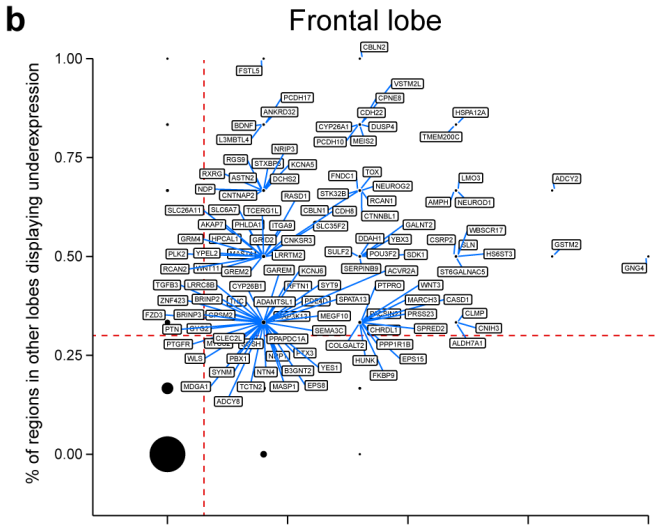

d

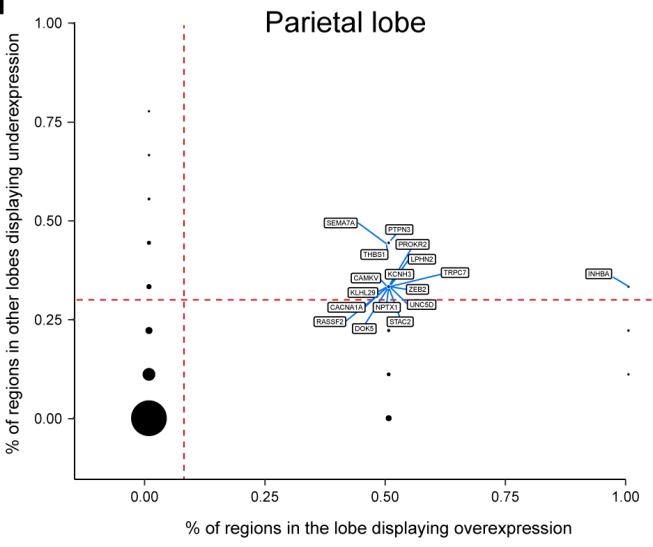

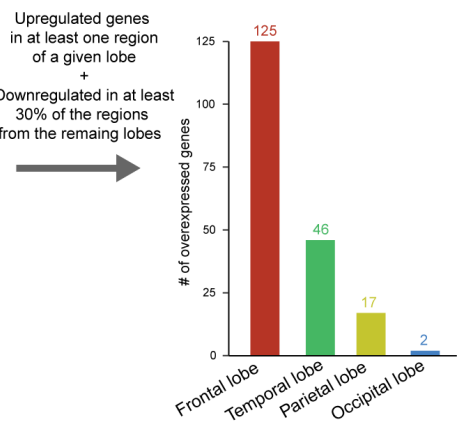

Temporal lobe

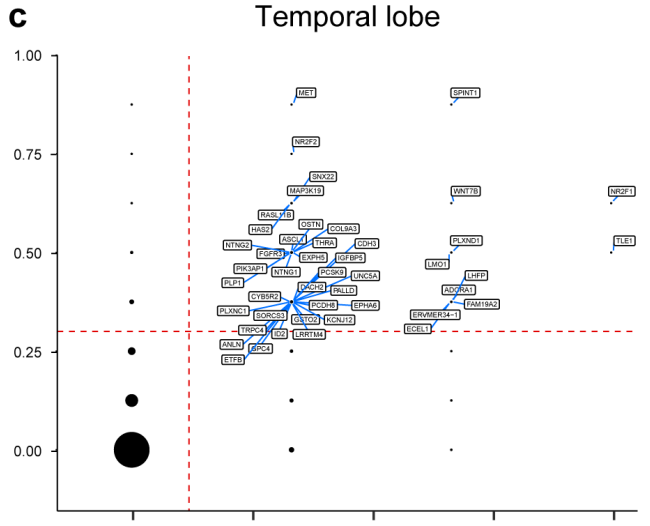

e

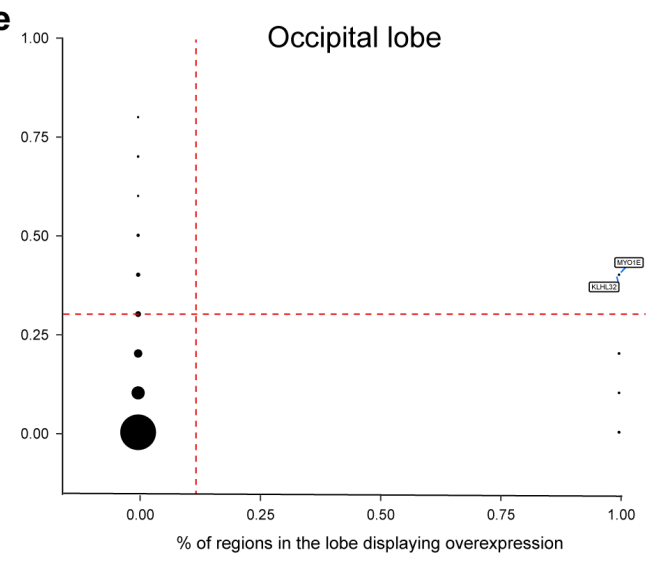

Extended Data Figure 1. Workflow for analysis of mid-fetal human transcriptome data and genes upregulated in individual lobes. a, Human developmental dataset and workflow for analysis to identify genes upregulated in each cortical lobe. b, Genes upregulated in the frontal lobe in comparison to the other lobes. The X-axis represents proportion of regions in the frontal lobe in which the gene is significantly upregulated according to RNentropy. The $\mathrm{Y}$-axis represents proportion of regions in the other lobes in which the gene is significantly downregulated according to RNentropy. Upregulated genes, the ones delimited by orange dashed lines, are labeled. c, d, e, Same for temporal, parietal and occipital lobes, respectively. 
a

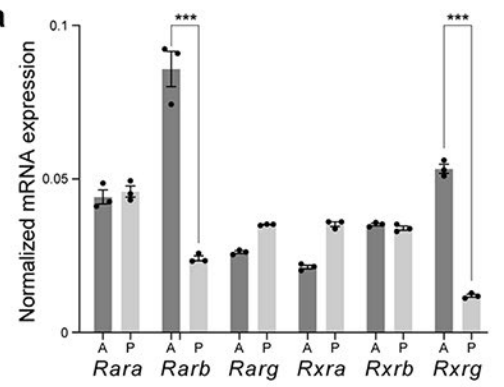

C

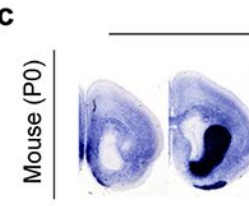

Rarb
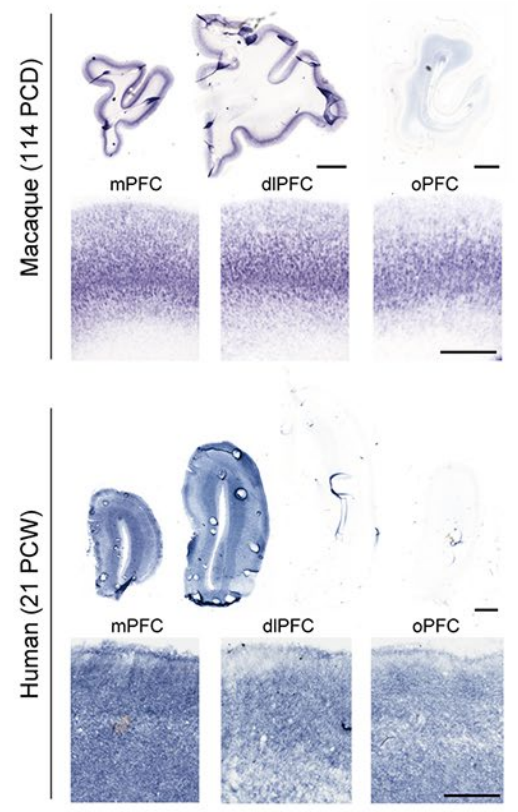

d

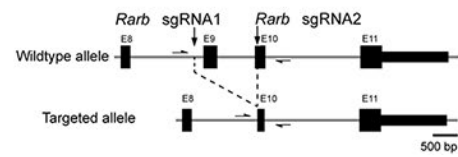

b

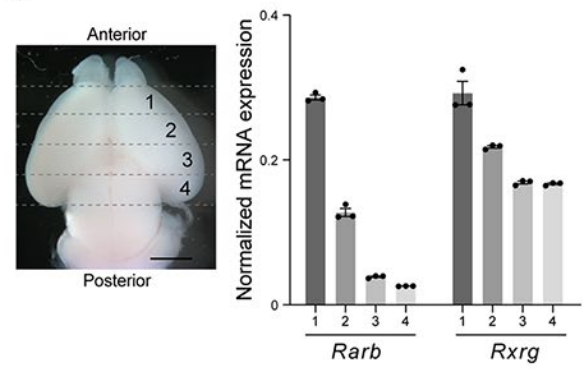

Rxrg
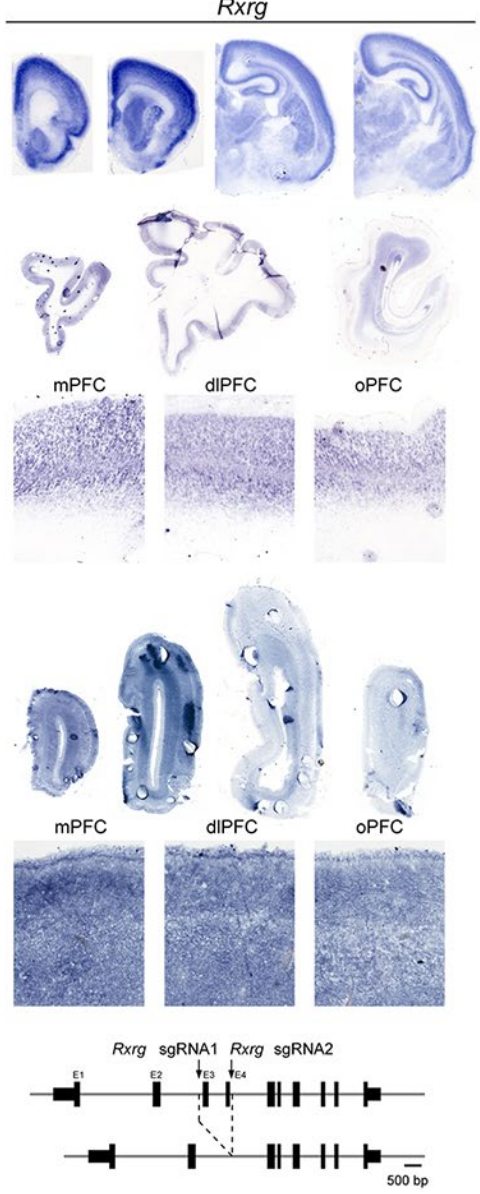

Extended Data Figure 2. RXRB and RARB are expressed in an anterior to posterior gradient. a, Quantitative PCR analysis of Rara, $b, g$ and Rxra, $b, g$ transcripts in the anterior and posterior half of mouse cortex at P0. Note that only expression of Rarb and Rxrg showed significant enrichment in the anterior cortex $\mathbf{b}$, Quantitative PCR analysis of Rxrg and Rarb transcripts in four sections dissected out of the cortical plate in anterior-posterior direction. Both genes showed an anterior-posterior gradient in expression level. Two-tailed Student's t-test: ${ }^{* * *} \mathrm{P}<0.0005 ; \mathrm{N}=3$ per condition; Errors bars: S.E.M. c, Expression of Rxrg and Rarb in mouse (P0), macaque (114 PCD) and human (21 PCW) brains by in situ hybridization. Higher magnification images of the regions of anterior cortex. Rxrg and Rarb transcripts are upregulated in the anterior part of the cortex in all three. Scale bars, $200 \mu \mathrm{m}$ (mouse); $2 \mathrm{~mm}$ (human); $500 \mu \mathrm{m}$ (human, higher magnification). $\mathrm{N}=2$ for human and macaque, $\mathrm{N}=3$ for mouse. $\mathrm{d}$, Strategies for the generation of $R \times r g$, and Rarb KO mice using CRISPR-Cas9 technique ${ }^{62}$. 


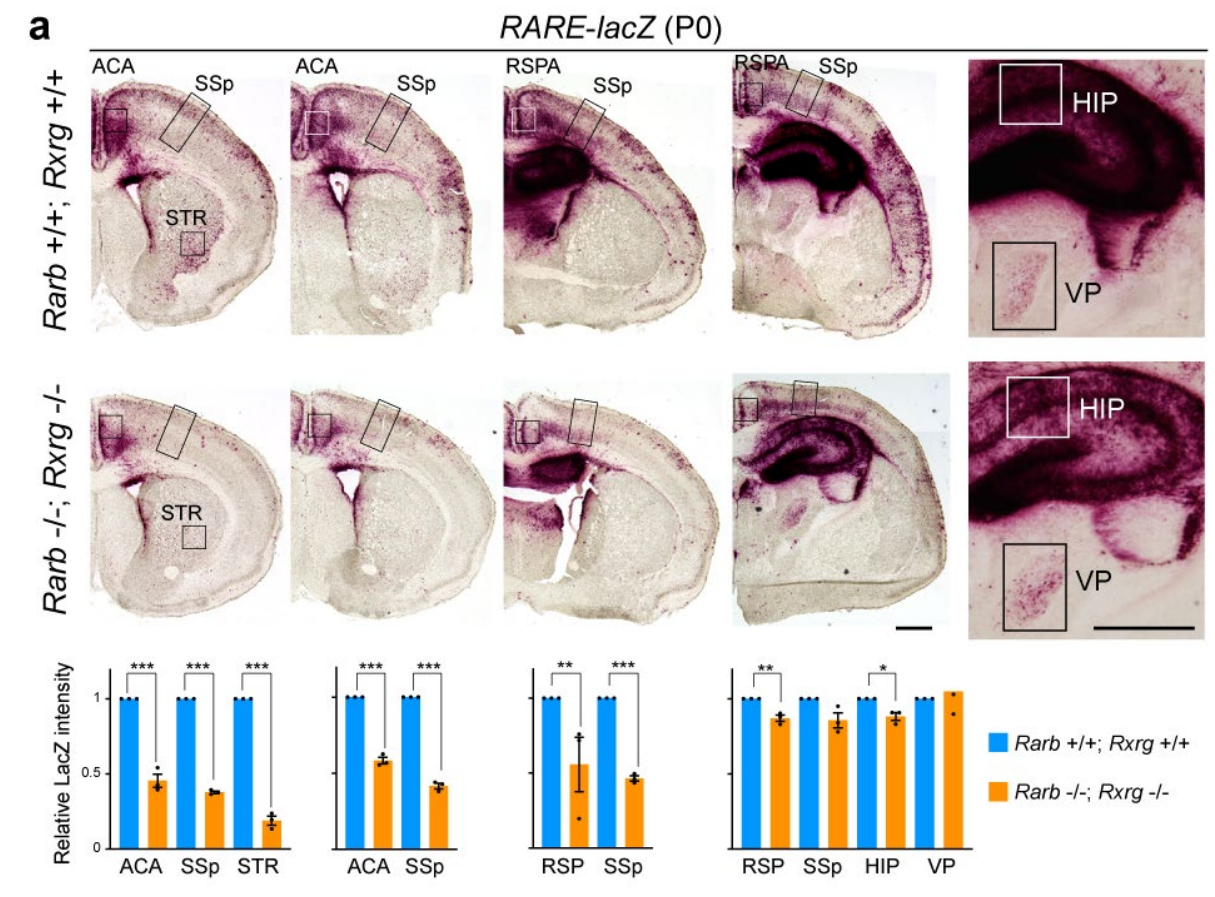

b

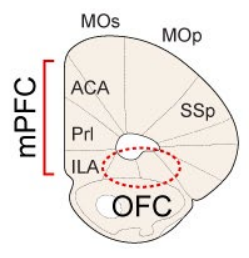

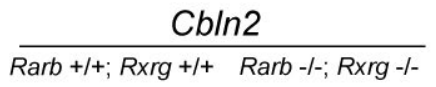

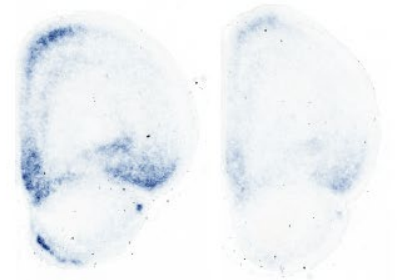

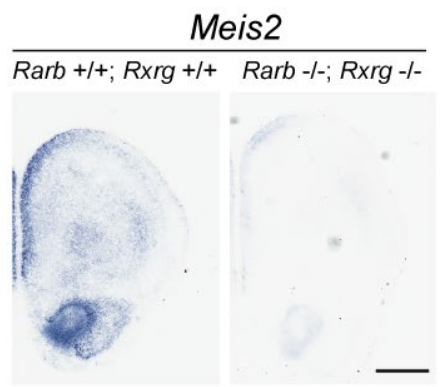

Extended Data Figure 3. Retinoic acid signaling in posterior regions of the P0 mouse forebrain. a, $\beta$-Galactosidase histochemical staining of more posterior regions of Rxrg+/+; Rarb+/+ (WT); RARE-lacZ and Rxrg-/-; Rarb-/- (dKO); RARE-lacZ mouse brains at P0. Signal intensity in the boxed area (ACA, SSp, RSPA, STR, HIP and VP) was quantified. Note the reduced activity in anteromedial structures including ACA and RSPA (RSP). There is also reduced expression in hippocampus and lateral striatum, but not the thalamus. Two-tailed Student's t-test; ${ }^{*} \mathrm{P}=0.009,{ }^{* *} \mathrm{P}<0.005,{ }^{* * *} \mathrm{P}<0.0005$; $\mathrm{N}=3$ per genotype: Errors bars: S.E.M.; Scale bars, $200 \mu \mathrm{m}$. b, Cbln2 and Meis2 expression in PO WT and dKO mutant brain by in situ hybridization at P0. Note that Cbln2 and Meis2 expression in mPFC was decreased in dKO. Scale bar, $200 \mu \mathrm{m}$. N = 3 per genotype. ACA, anterior cingulate area; HIP, hippocampus; RSPA/RSP, retrosplenial area; STR, striatum; VP, ventroposterior thalamus. 

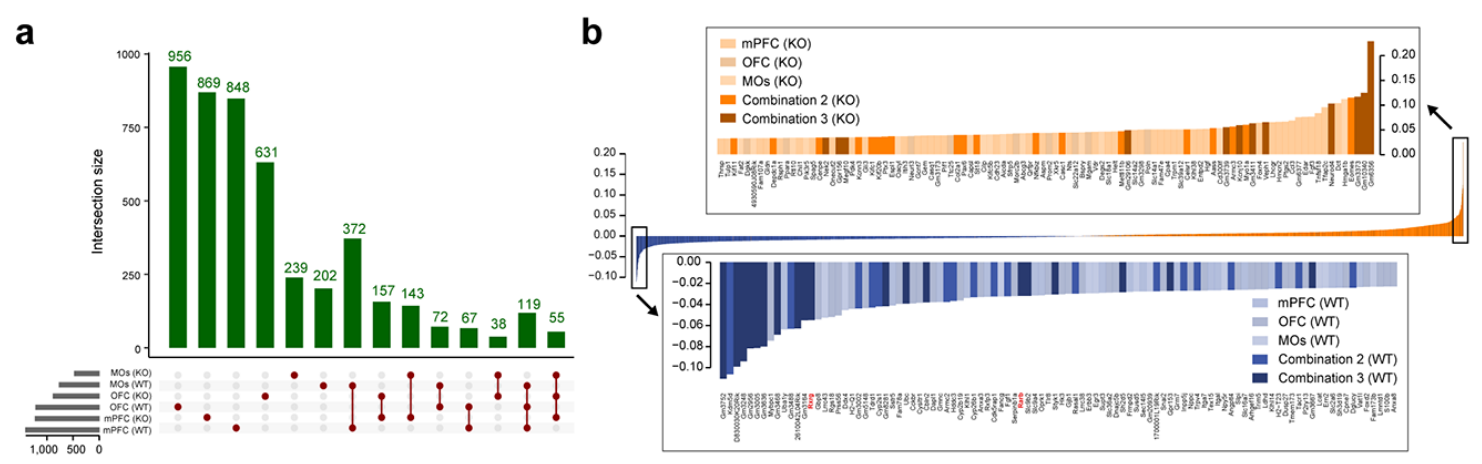

C

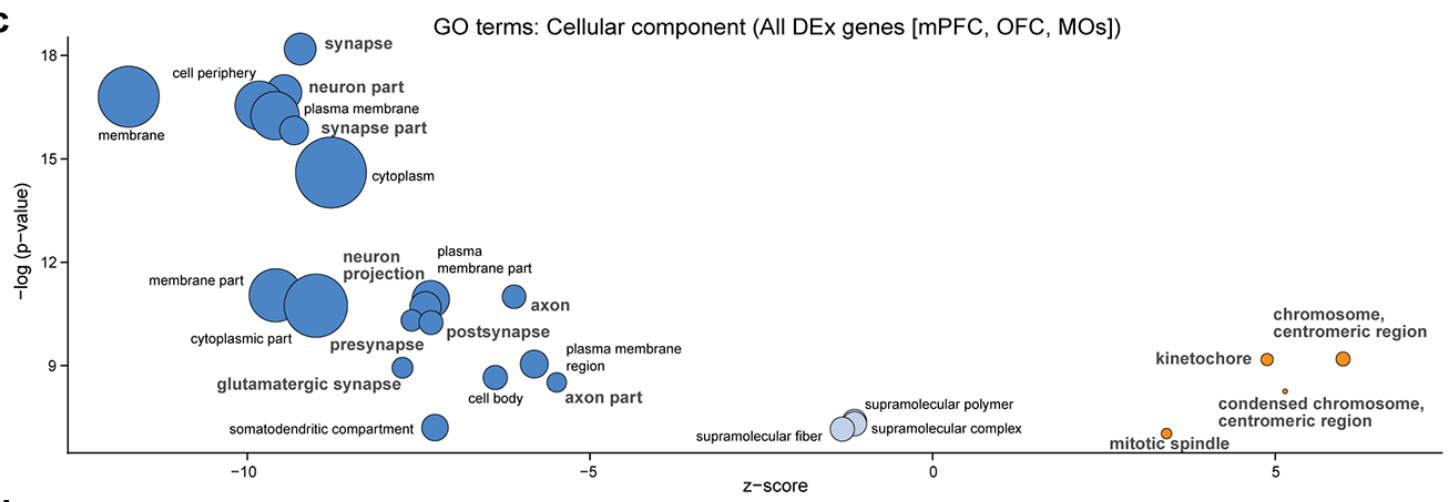

d DEx genes shared in MPFC, OFC, MOs DEx genes only in mPFC

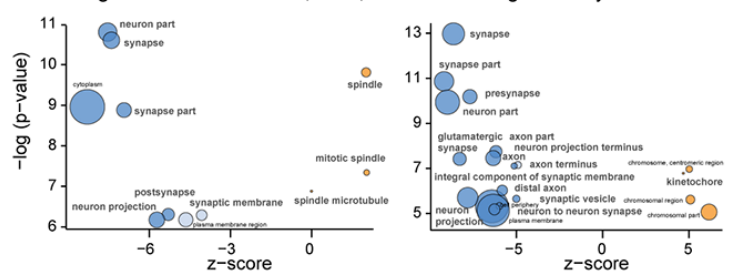

DEx genes only in OFC

DEx genes only in MOs

e
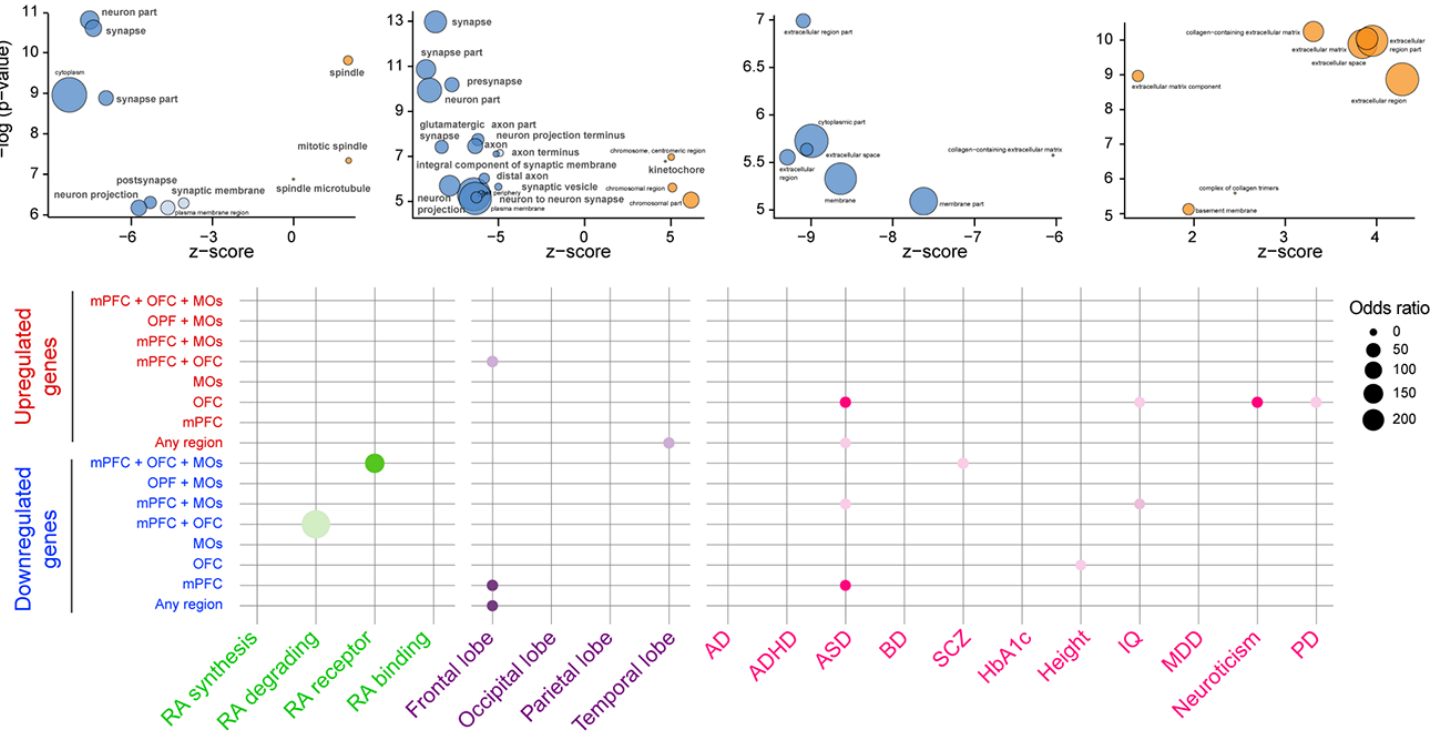

Extended Data Figure 4. Additional analysis of RNA-seq experiments. a, Number of upregulated genes between PO WT and dKO mice per region and phenotype, as well as combinations of regions and phenotypes. $\mathbf{b}$, Gene loadings of the first principal component from PCA in Fig. 3. Colors represent the frontal cortex region where the gene was found to be upregulated. c, Cellular component GO terms associated with the total list of 4,768 DEx genes found, showing their Z-score and nominal P values. Zscore represents the proportion of upregulated versus downregulated genes in the list of DEx genes associated to each GO term (i.e. z-score = (\#up - \#down) / sqrt (\#all DEx associated to the $G O$ term)). Dark blue: $z$-score $<-5$; light blue: $z$-score $(-5,0]$; orange: 1176 $z$-score $>0$. Size of the bubbles are proportional to the total number of DEx genes associated to the given GO term. $\mathbf{d}$, Cellular component GO terms associated with DEx 
1177 genes found in all three frontal cortex regions, and DEx genes unique to each region 1178 (mPFC, OFC, MOs) and their nominal P value. e, Enrichment of RA related genes 1179 (green), genes upregulated in individual lobes of the mid-fetal human cortex based on 1180 Fig. 1 (purple), and psychiatric disease related genes in up- and downregulated genes 1181 (pink). DEx genes are separated by genes that are DEx only in the given region (mPFC, 1182 OFC, MOs), genes that are DEx in the two given regions (mPFC + OFC, mPFC + MOs, 1183 OFC + MOs), genes that are DEx in all three regions (mPFC + OFC + MOs). Circles 1184 plotted for significant enrichments ( $P$ value $<0.05)$, in darker color, significance is 1185 considering the adjusted $\mathrm{P}$ value. Diameter of circle is associated with odds ratio per 1186 legend. 

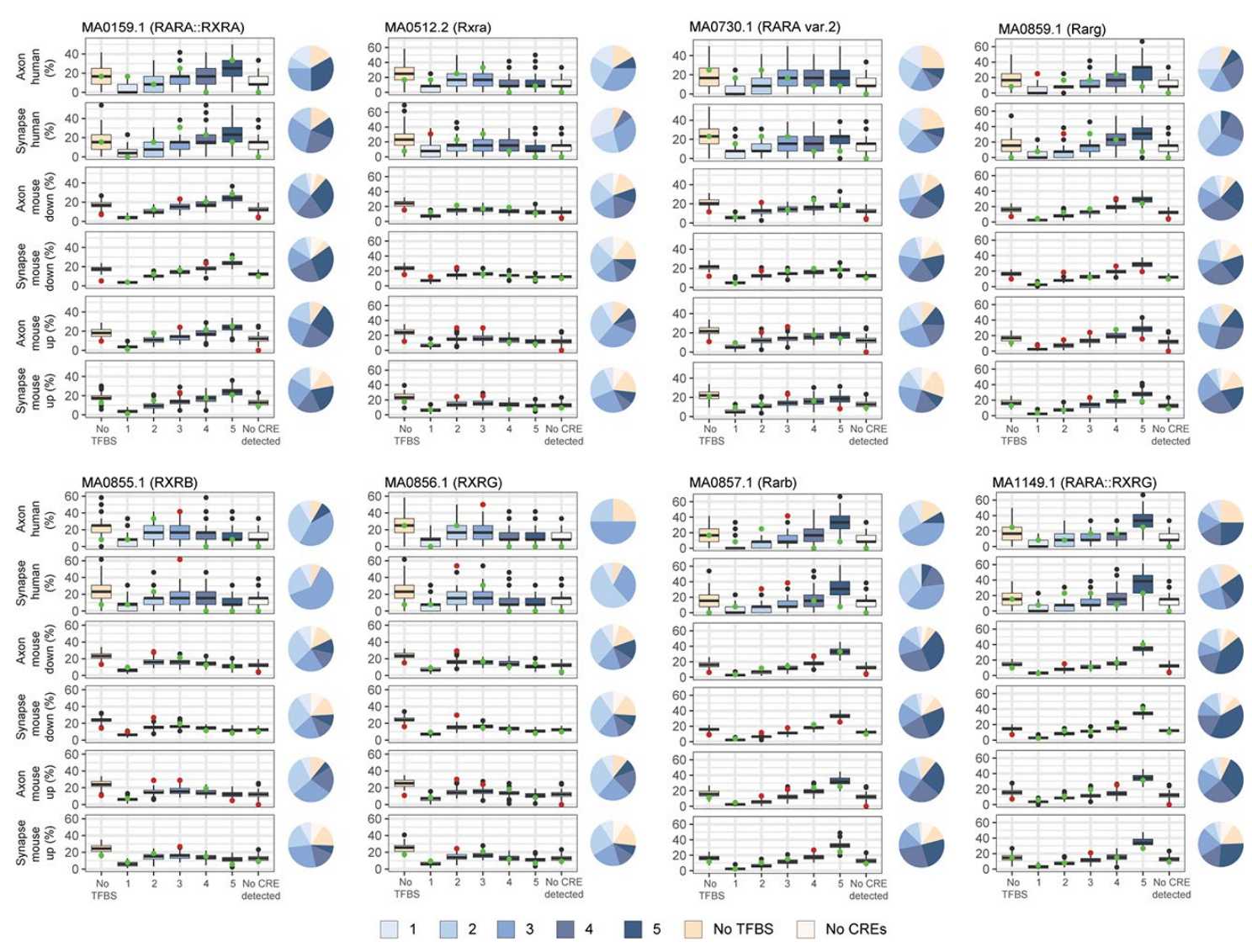

Extended Data Figure 5. Analysis of retinoic acid receptor binding sites in regulatory regions of upregulated synaptic and axon development genes. Boxplot showing the distribution of the proportion of genes in each gene set in categories representing different levels of sequence matches for each of 8 JASPAR RA receptors binding motifs. One to five represents distribution quantiles $(0.2,0.4,0.6,0.8,1)$ of the fraction of bases covered by RA binding sites in the union of the six gene lists. Category "No cis-regulatory element (CRE) detected" refers to genes with which we could not associate any human dIPFC/DFC mid-fetal H3K27ac peak. Colored dots represent the observed value for each category; red and green indicates whether they fall outside or inside the $95 \%$ confidence interval, respectively. Pie charts represent the observed proportion of genes in each category for each gene list and motif. 
a
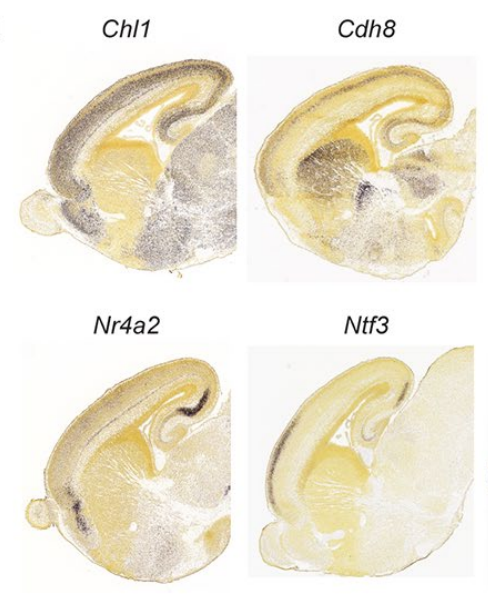
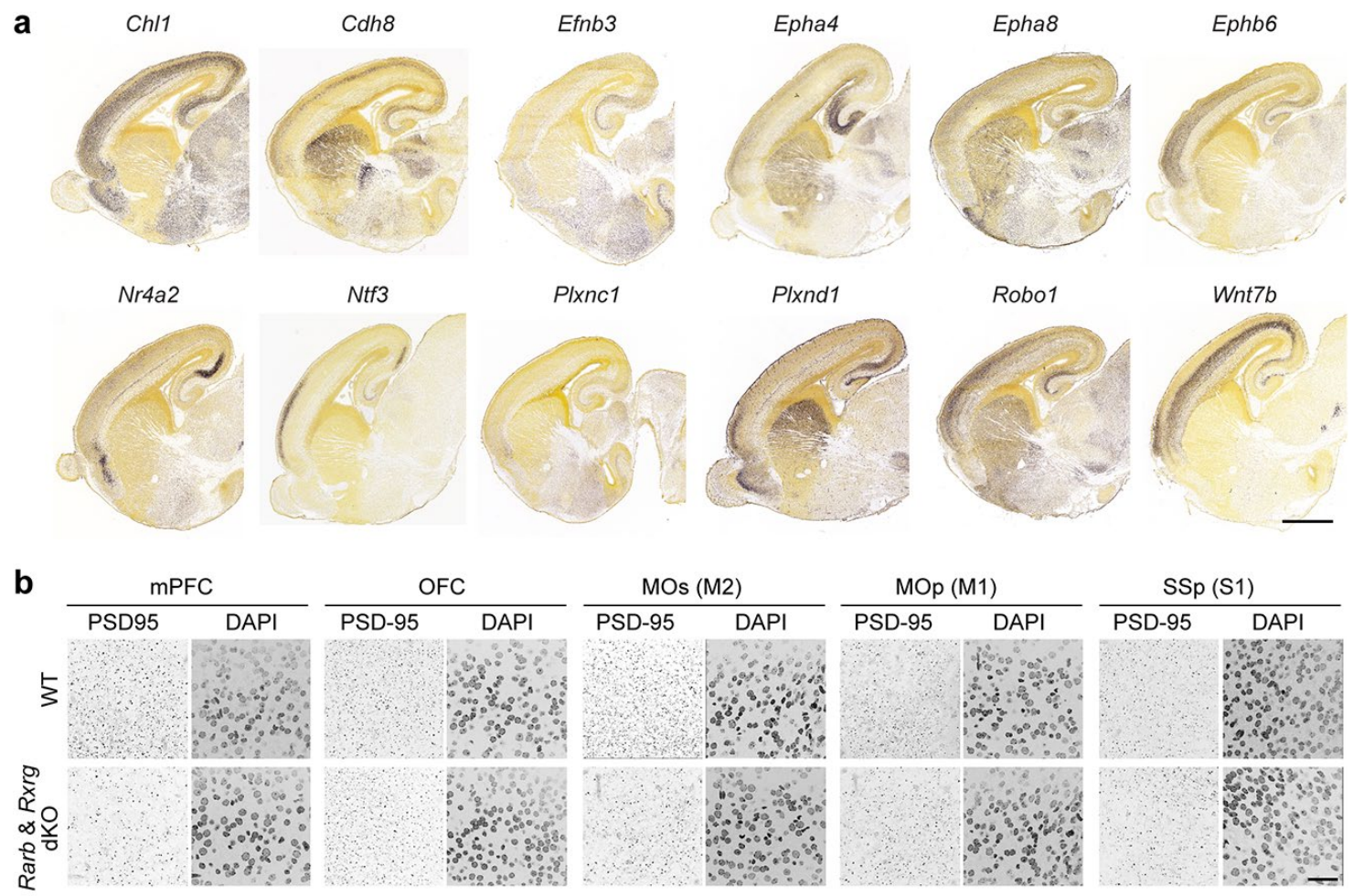

c
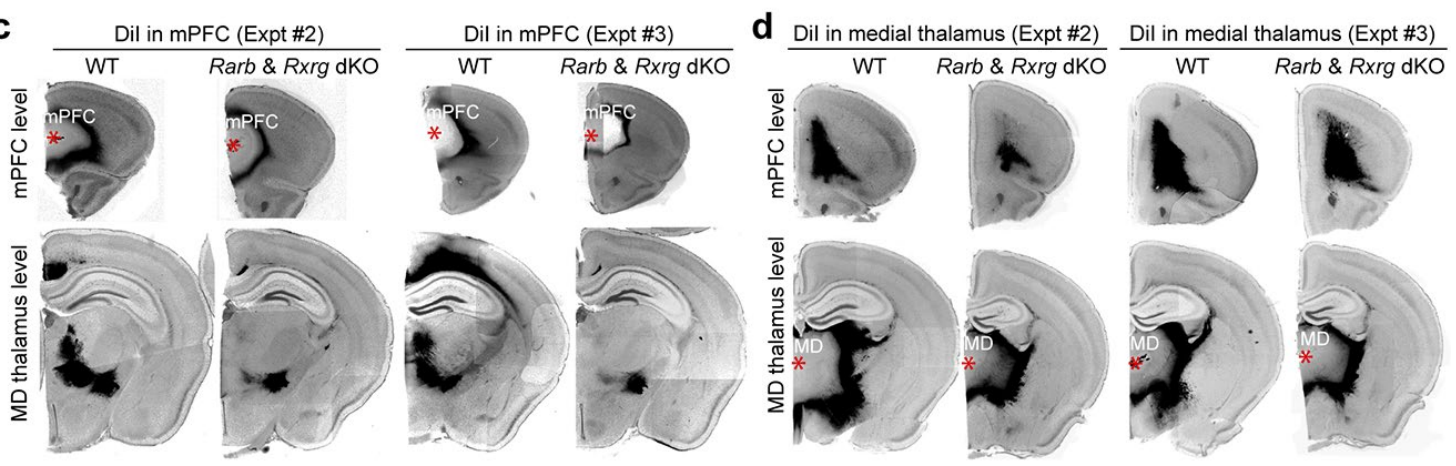

Extended Data Figure 6. Altered synaptic density and axonal projections in Rxrg/Rarb dKO mice. a, Example of downregulated genes between PO Rxrg+/+; Rarb+/+ (WT) and Rxrg-/-; Rarb-/- (dKO) that displayed an anterior to posterior gradient in 18 PCD mouse embryo (images are from the Allen Developing Mouse Brain Atlas; developingmouse.brain-map.org/). Scale bar: $1 \mathrm{~mm}$. b, Immunostaining for PSD95/DLG4 in the cortical subregions (mPFC, MOs, OFC, MOp, and SSp) of WT and dKO brain at P0. Each region as shown in Figure 2c. Scale bar: $25 \mu \mathrm{m}$. N = 3 per genotype. c,d, Dil placement in mPFC (c) and medial thalamus (d) with tracing data in WT and $\mathrm{dKO}$ brain at P21. Additional two replicates of experiment shown in Figure 4d,e are shown. Asterisks: Dil crystal placement. Scale bar: $1 \mathrm{~mm}$. 

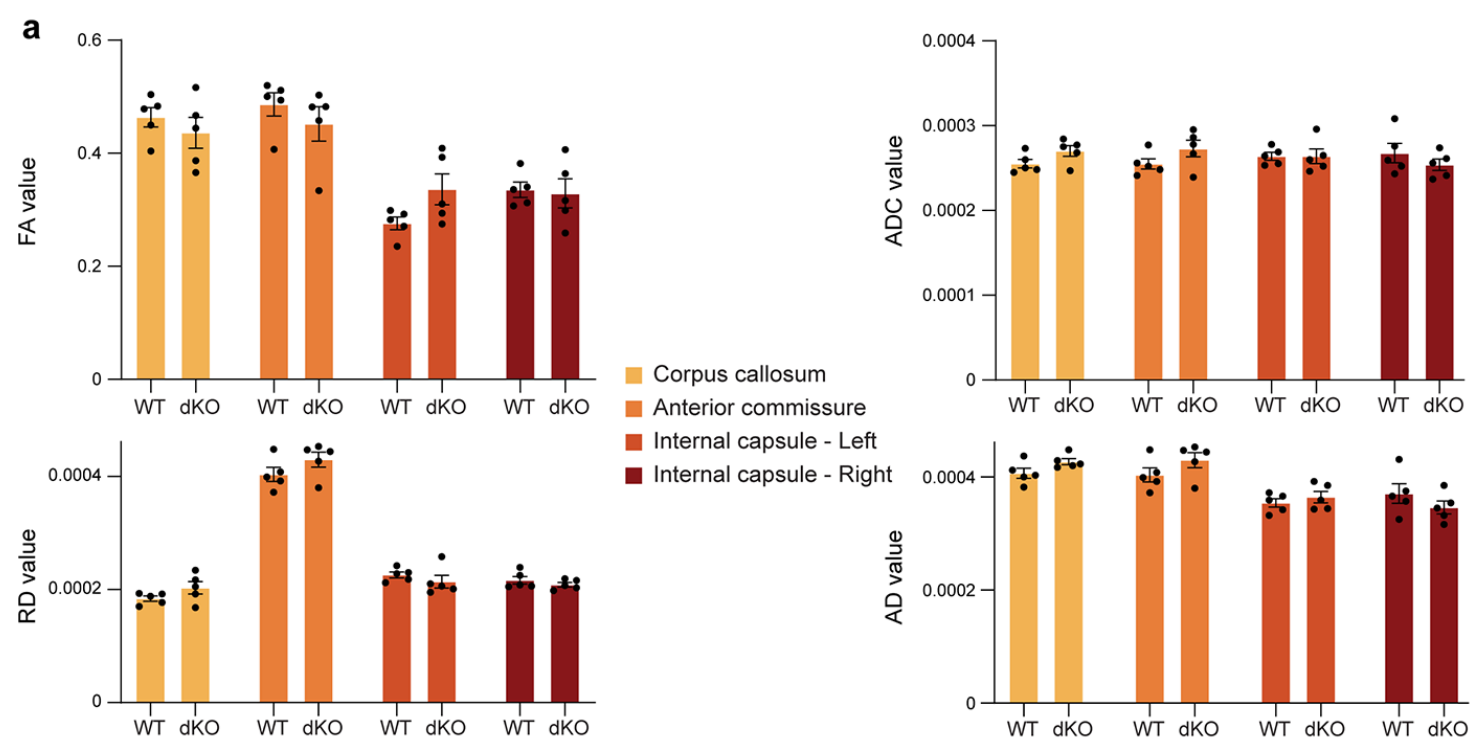

b
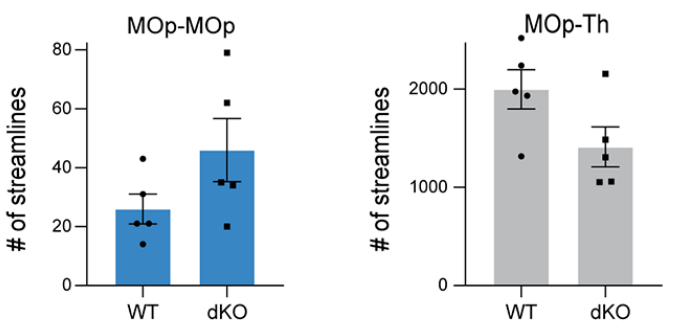

Internal capsule - Left

- Internal capsule - Right
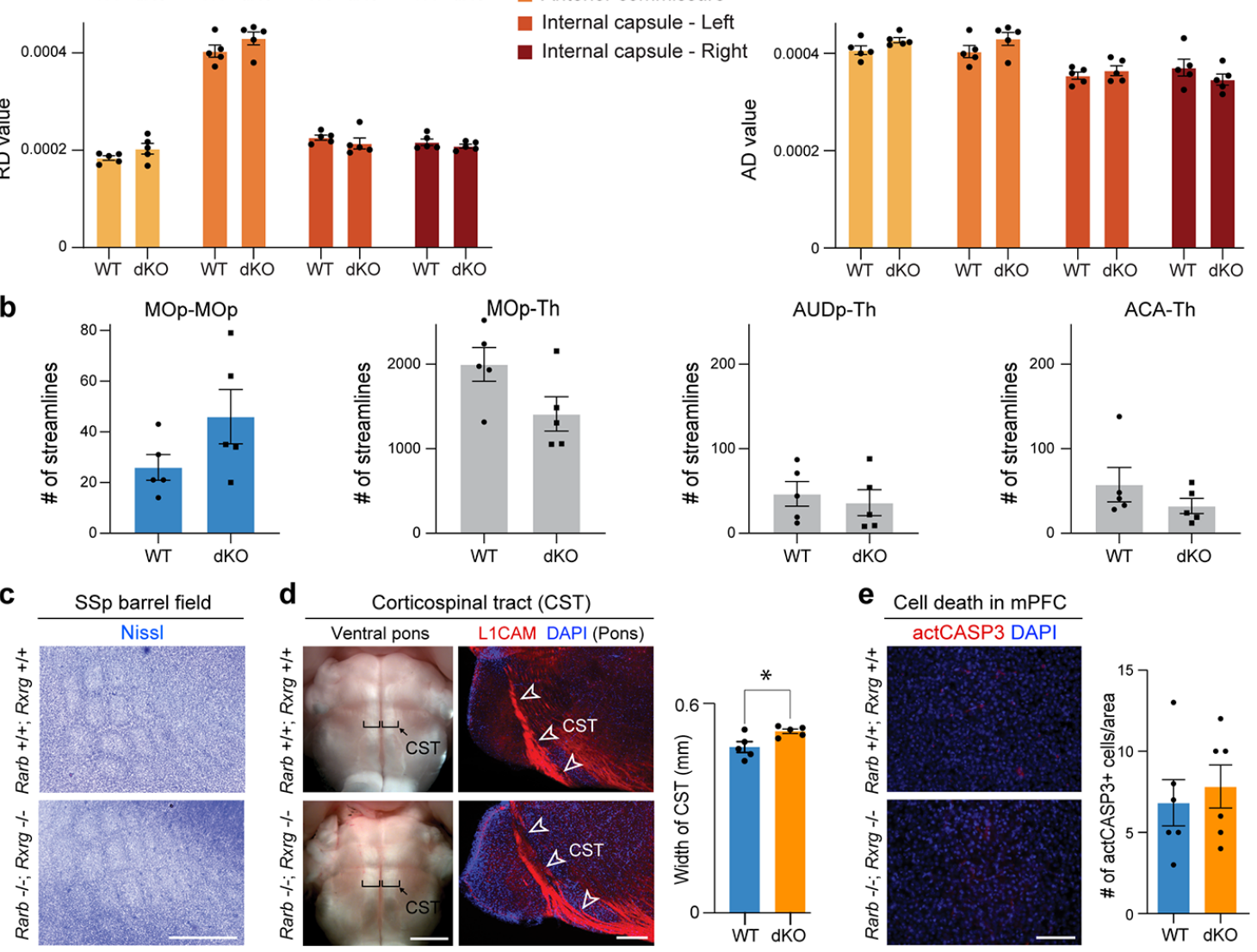

Extended Data Figure 7. Analysis of axonal projections and cell death in

RxrgIRarb dKO mice. a, Four scalar indexes which describe microstructural integrity do not differ in the four major white-matter tracts (corpus callosum, anterior commissure, left and right internal capsules) between WT and dKO mice. b, Number of streamlines of MOp-thalamus and corticocortical tracts, did not differ between WT and dKO. Paired t-test: NS; N = 5 per genotype; Errors bars: S.E.M. c, Barrel field formation was grossly normal in dKO. Barrel formation was examined by Nissl staining at P5. $\mathrm{N}=3$ per genotype. d, Corticospinal tract (CST) appearance at P30 was grossly normal in dKO. The width of the corticospinal tract (shown in brackets) is slightly increased in dKO. Sagittal section at P5 was stained with anti-L1CAM antibody to show the grossly normal CST formation in dKO. Two-tailed Student's t-test: * $\mathrm{P}=0.03 ; \mathrm{N}=5$ per genotype; Errors bars: S.E.M. e, Frequency of apoptosis in the mPFC detected by cleaved caspase 3 (actCASP3) between WT and dKO is not significantly different. Two-tailed Student's t1283 test: WT vs. dKO; NS; N = 5 per genotype; Errors bars: S.E.M.; Scale bars: $500 \mu \mathrm{m}$; $200 \mu \mathrm{m}$ (b); $1 \mathrm{~mm}$ (c); $100 \mu \mathrm{m}(\mathrm{d})$. 

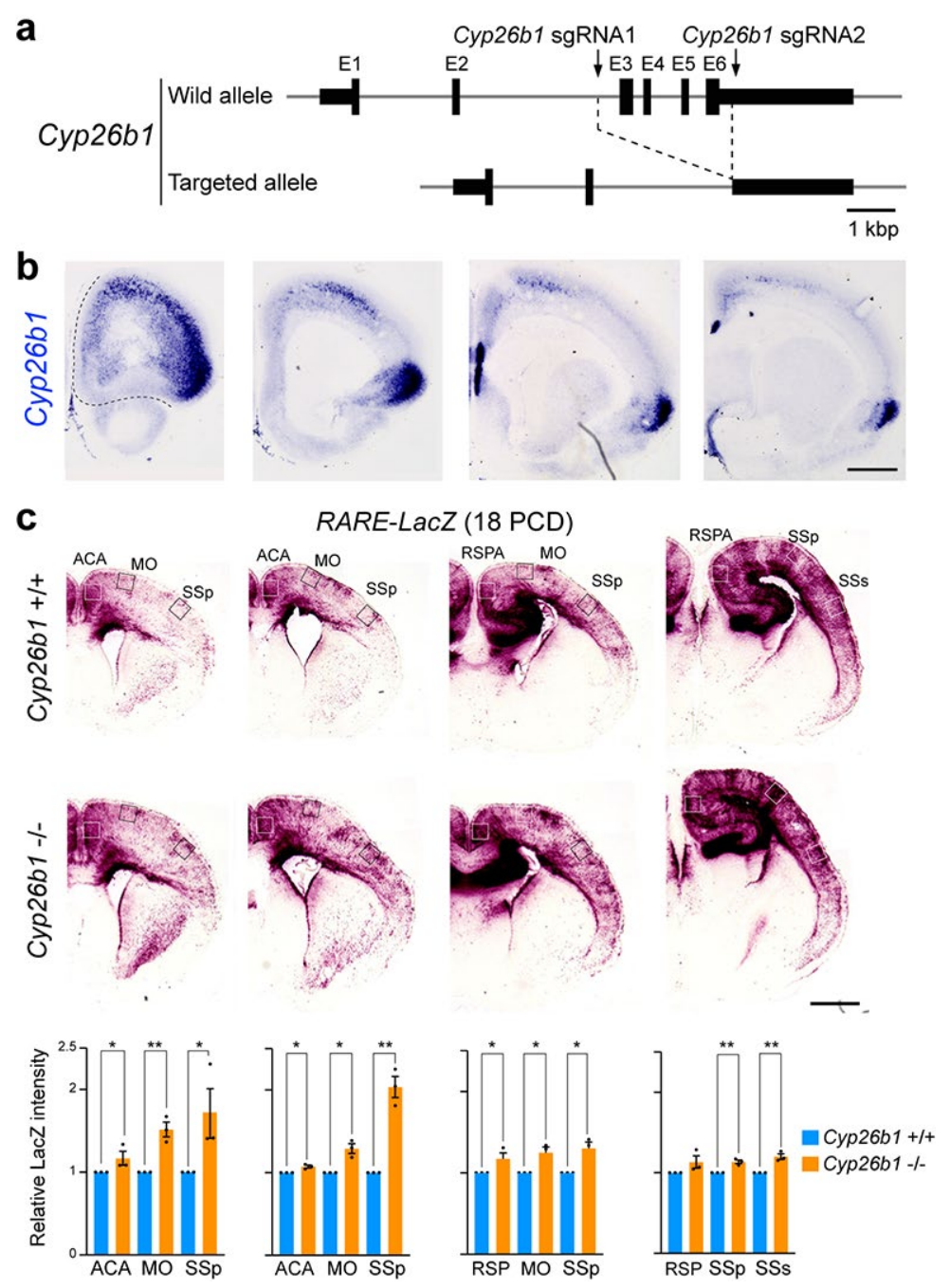

Extended Data Figure 8. Retinoic acid signal in posterior cortical regions of Cyp26b1 KO mice. a, Strategy for the generation of Cyp26b1-/- (Cyp26b1 KO) mice using CRISPR-Cas9 gene editing technique ${ }^{62}$. b, Cyp26b1 expression in P0 mice cortex by in situ hybridization. The colorimetric staining was purposefully extended compared to the experiment in Fig. 5a to better visualize low expressing locations. c, $\beta$ Galactosidase staining of more posterior regions of control Cyp26b1+/+; RARE-lacZ (Ctrl) and Cyp26b1-I-; RARE-lacZ (KO) mouse brains at 18 PCD. Scale bar: $500 \mu \mathrm{m}$. Intensity of signal in the boxed areas (ACA, MO, SSp) was quantified. Increase in RA signaling in Cyp26b1 KO brains is less significant in posterior regions. Two-tailed Student's t-test: Ctrl vs. Cyp26b1 KO: ${ }^{*} \mathrm{P}<0.05,{ }^{*} \mathrm{P}<0.005, \mathrm{~N}=3$ per genotype; Errors bars: S.E.M.; Scale bars: $200 \mu \mathrm{m}$. 
a

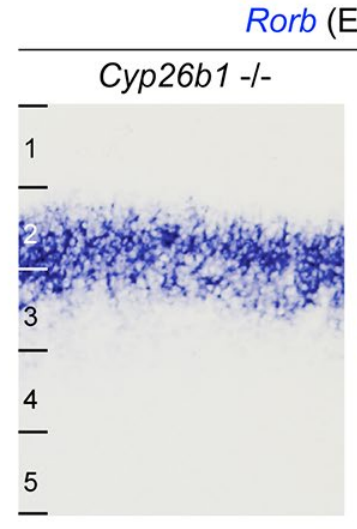

Rorb (Expt \#2)

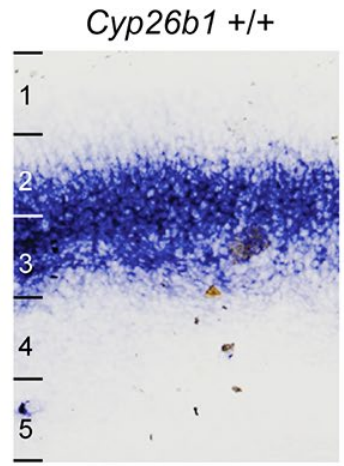

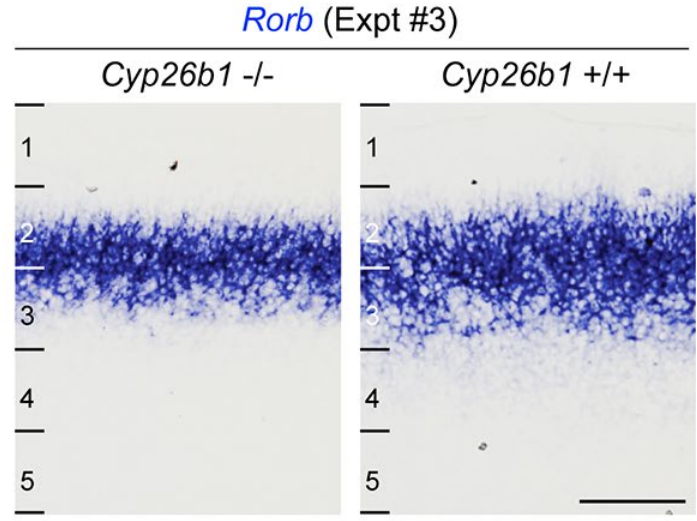

Dil placement in thalamus

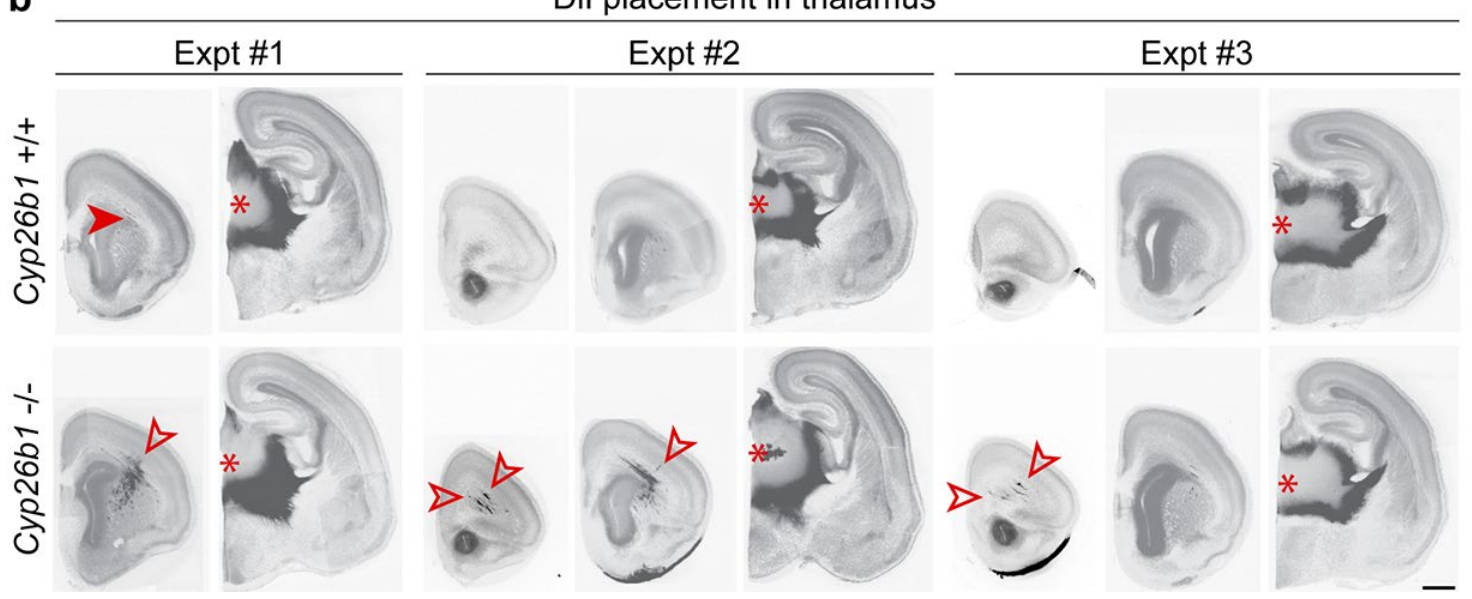

\section{Extended Data Figure 9. Rorb expression and thalamocortical projections in the} perinatal Cyp26b1 KO mice. a, 18 PCD, Cyp26b1 KO mouse brains show upregulation of Rorb. Additional two replicates are shown. Scale bar: $100 \mu \mathrm{m}$. b. Dil was placed in the medial thalamus of WT and Cyp26b1 KO brains, and signal was detected in the PFC. Additional two replicates of experiment in Figure $5 \mathrm{c}$ are shown. N $=3$ per genotype and condition. Arrowheads: Thalamocortical innervation of the medial and dorso-lateral frontal cortex. Asterisks: Dil crystals placed. Scale bar: $400 \mu \mathrm{m}$. 
a

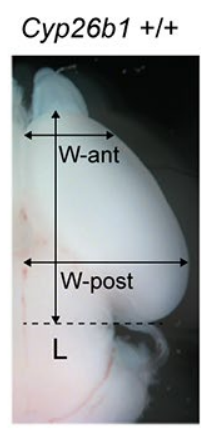

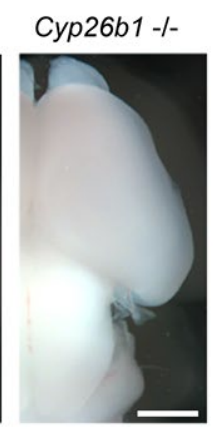

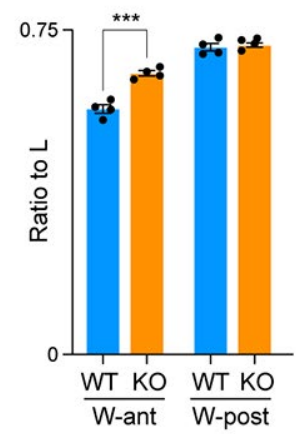

b
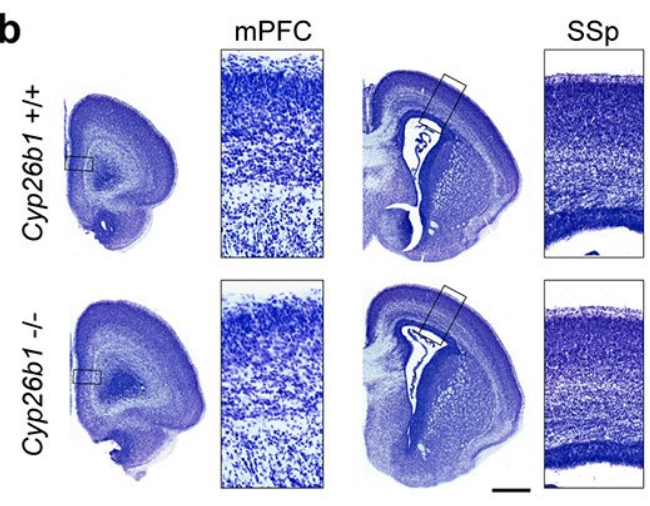

C
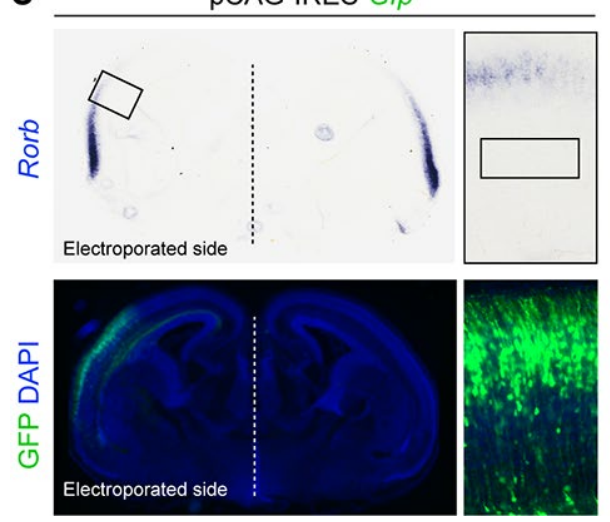
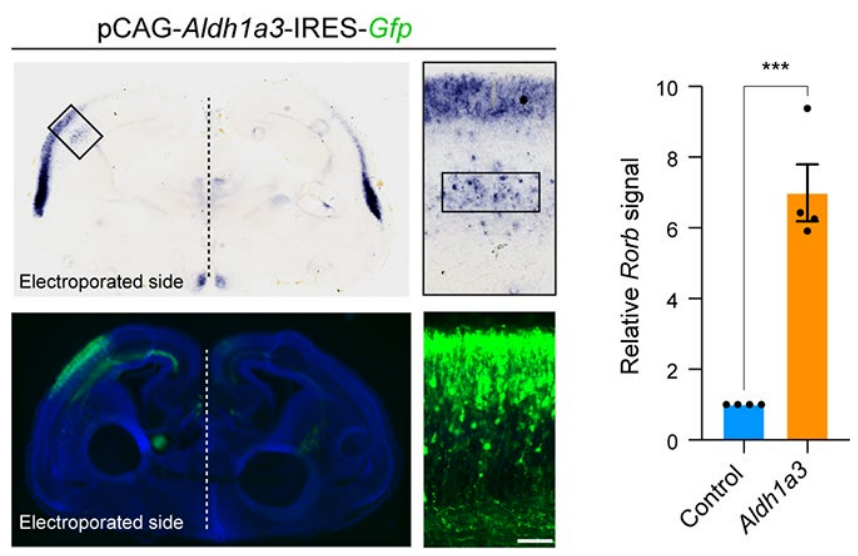

Extended Data Figure 10. Ectopic RA signaling leads to enlargement of frontal cortex and expanded Rorb expression. a, Cyp26b1 KO brain at 18 PCD showed enlargement of anterior/frontal cortex and not posterior cortex. Ratio of brain width at anterior cortex to total brain length was significantly increased in Cyp26b1 KO brain compared WT. Two-tailed Student's t-test: WT vs. Cyp26b1 KO: ${ }^{* *} \mathrm{P}=0.0001 ; \mathrm{N}=4$ per genotype; Errors bars: S.E.M. b. Nissl staining reveals that the cortical wall and cortical plate are grossly normal when analyzed in the mPFC and SSp of Cyp26b1 KO. c, Electroporation of either control pCAG-IRES-Gfp or pCAG-Aldh1a3-IRES-Gfp expression vector plasmid in the dorso-lateral fronto-parietal wall at 14 PCD. Brains were dissected out at P5. Misexpression of Aldh1a3 leads to regional expansion and ectopic laminar expression of Rorb, when assessed by in situ hybridization. GFP expression as a marker of misexpressing cells are shown in lower panels. Rorb signal intensity in the boxed area in the cortex was quantified. Two-tailed Student's t-test: ${ }^{* * *} P$ $=0.0001 ; \mathrm{N}=4$ per genotype; Errors bars: S.E.M.; Scale bars: $500 \mu \mathrm{m}$ (a); $200 \mu \mathrm{m}$ (b); 